\title{
SRI Education
}

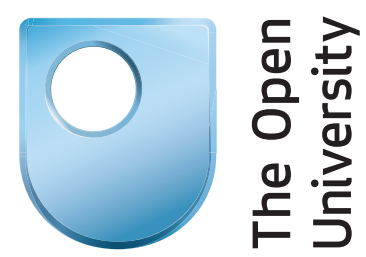

\section{Innovating Pedagogy 2015}

Exploring new forms of teaching, learning and assessment, to guide educators and policy makers

Mike Sharples, Anne Adams, Nonye Alozie, Rebecca Ferguson, Elizabeth FitzGerald, Mark Gaved, Patrick McAndrew, Barbara Means, Julie Remold, Bart Rienties, Jeremy Roschelle, Kea Vogt, Denise Whitelock, Louise Yarnall

Open University Innovation Report 4

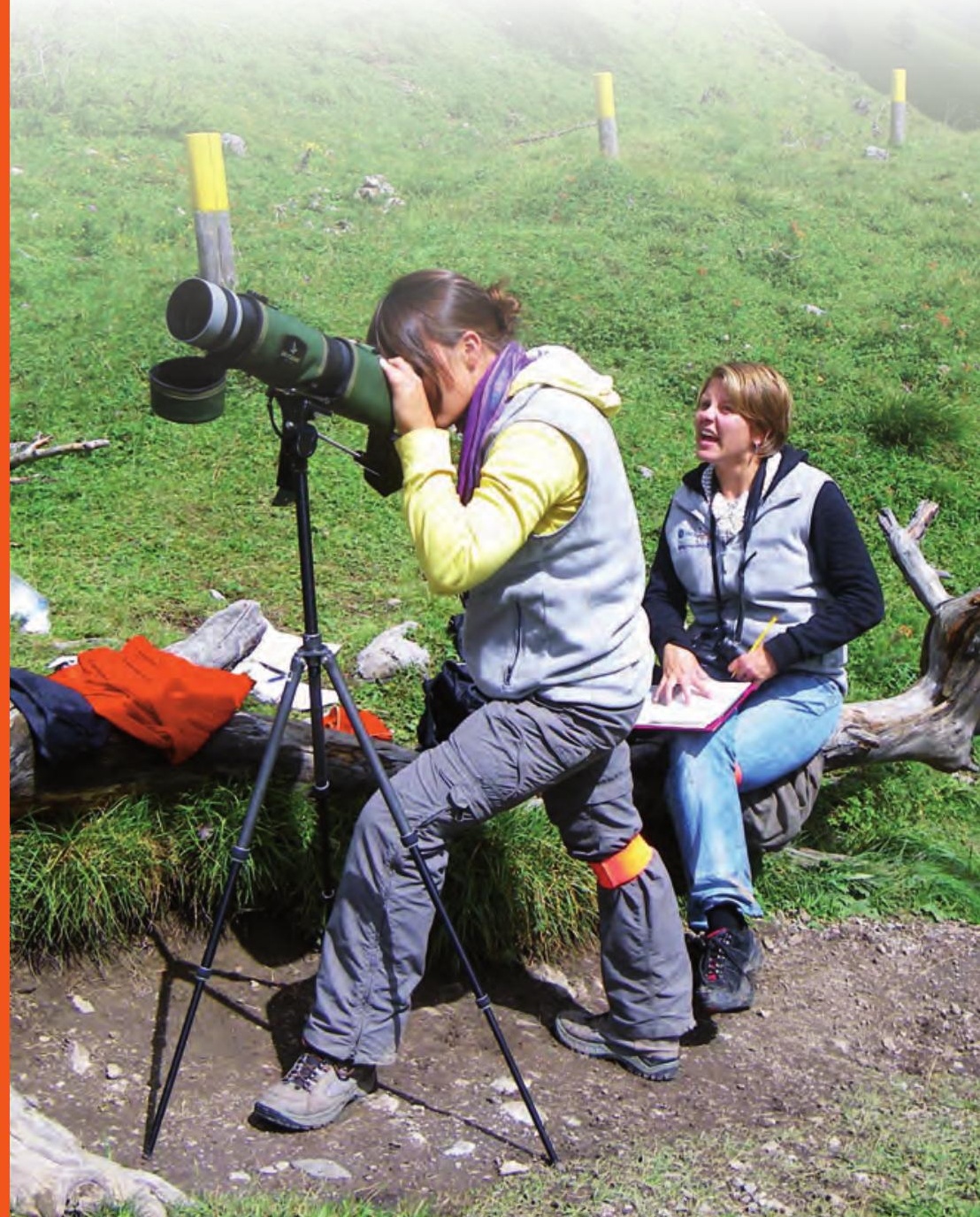


Permission is granted under a Creative Commons Attribution Licence to copy, redistribute, remix, transform and build upon this report freely, provided that attribution is provided as illustrated in the citation below. You may make changes in any reasonable manner, as long as you indicate that you have done this and do not imply that the licensor endorses you or your use. To view a copy of this licence, visit creativecommons.org/licenses/by/3.0

A full-text PDF version of this report is available to download from www.open.ac.uk/innovating

This material is based in part upon work supported by the National Science Foundation under Grant No. IIS-1233722. Any opinions, findings, and conclusions or recommendations expressed in this material are those of the author(s) and do not necessarily reflect the views of the National Science Foundation.

\section{Illustrations:}

Front-cover illustration of ESRI Geography Summer Camp @ C Christian Sailer, ETH Zürich. www.schulgis.ch/29401.html Reproduced with permission.

Page 12. Session by the Teacher Institute at the Exploratorium San Francisco on 'Reengineering your Science Curriculum'. Photograph by Gayle Laird (C) Exploratorium, www.exploratorium.edu Reproduced with permission.

Page 15. Argument visualisation in Cohere. Figure redrawn from Buckingham Shum, S. (2008). Cohere: Towards Web 2.0 Argumentation. In Proceedings of COMMA'08: 2nd International Conference on Computational Models of Argument, 28-30 May 2008, Toulouse, France. IOS Press, pp. 97-108.

Page 18. Children closely observe a banana slug during unstructured play. Photograph by Julie Remold. Reproduced with permission.

Page 21. Aris context-based game showing the campus location of a student protest in the 1960s. Image (C) Chris Holden, University of New Mexico. Reproduced with permission.

Page 24. Section of Scratch code for a hide-and-seek game.

Screen captured from scratch.mit.edu/projects/10128368/\#editor

Page 27. ARROW remotely controlled radio telescope for Open University students. From the OpenScience Laboratory learn5.open.ac.uk/course/format/sciencelab/section.php?name=af_sxpa288

Page 31. Children interacting with a tabletop display on the JuxtaLearn project. Image @ Anne Adams.

Reproduced with permission.

Page 34. Learner dashboard from the Cerego memorisation system.

Screen captured from cerego.com/sets/720261/learn

Page 36. Eyetracking study comparing people's ability to recognise emotion in real and virtual faces. Image (C) Christian Joyal. Reproduced with permission.

Page 39. Cover art for Portal 2 game. (C) Valve Corporation. www.thinkwithportals.com

\section{Suggested citation:}

Sharples, M., Adams, A., Alozie, N., Ferguson, R., FitzGerald, E., Gaved, M., McAndrew, P., Means, B., Remold, J., Rienties, B., Roschelle, J., Vogt, K., Whitelock, D. \& Yarnall, L. (2015). Innovating Pedagogy 2015: Open University Innovation Report 4. Milton Keynes: The Open University.

Institute of Educational Technology, The Open University, Walton Hall, Milton Keynes, MK7 6AA, United Kingdom

Center for Technology in Learning, SRI International, 333 Ravenswood Avenue, Menlo Park, CA 94025-3493, United States

(C) The Open University, 2015. ISBN 978-1-4730-2017-7 


\section{Contents}

Executive summary

\section{Introduction}

Crossover learning

Connecting formal and informal learning

Learning through argumentation

Developing skills of scientific argumentation

Incidental learning

Harnessing unplanned or unintentional learning

\section{Context-based learning}

How context shapes and is shaped by the process of learning

\section{Computational thinking}

Solving problems using techniques from computing

Learning by doing science with remote labs

Guided experiments on authentic scientific equipment

\section{Embodied learning}

Making mind and body work together to support learning

\section{Adaptive teaching}

Adapting computer-based teaching to the learner's knowledge and action

\section{Analytics of emotions}

Responding to the emotional states of students

Stealth assessment

Unobtrusive assessment of learning processes 


\section{Executive summary}

This series of reports explores new forms of teaching, learning and assessment for an interactive world, to guide teachers and policy makers in productive innovation. This fourth report proposes ten innovations that are already in currency and are having an increasing effect on education. To produce it, a group of academics at the Institute of Educational Technology in The Open University collaborated with researchers from the Center for Technology in Learning at SRI International. We proposed a long list of new educational terms, theories, and practices. We then pared these down to ten that have the potential to provoke major shifts in educational practice, particularly in post-school education. Lastly, we drew on published and unpublished writings to compile the ten sketches of new pedagogies that might transform education. These are summarised below in an approximate order of immediacy and timescale to widespread implementation.
Crossover learning Learning in informal settings, such as museums and after-school clubs, can link educational content with issues that matter to learners in their lives. These connections work in both directions. Learning in schools and colleges can be enriched by experiences from everyday life; informal learning can be deepened by adding questions and knowledge from the classroom. These connected experiences spark further interest and motivation to learn. An effective method is for a teacher to propose and discuss a question in the classroom, then for learners to explore that question on a museum visit or field trip, collecting photos or notes as evidence, then share their findings back in the class to produce individual or group answers. These crossover learning experiences exploit the strengths of both environments and provide learners with authentic and engaging opportunities for learning. Since learning occurs over a lifetime, drawing on experiences across multiple settings, the wider opportunity is to support learners in recording, linking, recalling and sharing their diverse learning events.

Learning through argumentation Students can advance their understanding of science and mathematics by arguing in ways similar to professional scientists and mathematicians. Argumentation helps students attend to contrasting ideas, which can deepen their learning. It makes technical reasoning public, for all to learn. It also allows students to refine ideas with others, so they learn how scientists work together to establish or refute claims. Teachers can spark meaningful discussion in classrooms by encouraging students to ask open-ended questions, re-state remarks in more scientific language, and develop and use models to construct explanations. When students argue in scientific ways, they learn how to take turns, listen actively, and 
respond constructively to others. Professional development can help teachers to learn these strategies and overcome challenges, such as how to share their intellectual expertise with students appropriately.

Incidental learning Incidental learning is unplanned or unintentional learning. It may occur while carrying out an activity that is seemingly unrelated to what is learned. Early research on this topic dealt with how people learn in their daily routines at their workplaces. For many people, mobile devices have been integrated into their daily lives, providing many opportunities for technology-supported incidental learning. Unlike formal education, incidental learning is not led by a teacher, nor does it follow a structured curriculum, or result in formal certification. However, it may trigger self-reflection and this could be used to encourage learners to reconceive what could otherwise be isolated learning fragments as part of more coherent and longer term learning journeys.

Context-based learning Context enables us to learn from experience. By interpreting new information in the context of where and when it occurs and relating it to what we already know, we come to understand its relevance and meaning. In a classroom or lecture theatre, the context is typically confined to a fixed space and limited time. Beyond the classroom, learning can come from an enriched context such as visiting a heritage site or museum, or being immersed in a good book. We have opportunities to create context, by interacting with our surroundings, holding conversations, making notes, and modifying nearby objects. We can also come to understand context by exploring the world around us, supported by guides and measuring instruments. It follows that to design effective sites for learning, at schools, museums and websites, requires a deep understanding of how context shapes and is shaped by the process of learning.

Computational thinking Computational thinking is a powerful approach to thinking and problem solving. It involves breaking large problems down into smaller ones (decomposition), recognizing how these relate to problems that have been solved in the past (pattern recognition), setting aside unimportant details (abstraction), identifying and developing the steps that will be necessary to reach a solution (algorithms) and refining these steps (debugging). Such computational thinking skills can be valuable in many aspects of life, ranging from writing a recipe to share a favourite dish with friends, through planning a holiday or expedition, to deploying a scientific team to tackle a difficult challenge like an outbreak of disease. The aim is to teach children to structure problems so they can be solved. Computational thinking can be taught as part of mathematics, science and art or in other settings. The aim is not just to encourage children to be computer coders, but also to master an art of thinking that will enable them to tackle complex challenges in all aspects of their lives.

Learning by doing science with remote labs Engaging with authentic scientific tools and practices such as controlling remote laboratory experiments or telescopes can build science inquiry skills, improve conceptual understanding, and increase motivation. Remote access to specialized equipment, first developed for scientists and university students, is now expanding to trainee teachers and school students. A remote lab typically consists of apparatus or equipment, robotic arms to operate it, and cameras that provide views of the experiments as they unfold. Remote lab systems can reduce barriers to participation by providing user-friendly Web interfaces, curriculum materials, and professional development for teachers. With appropriate support, access to remote labs can deepen understanding for teachers and students by offering hands-on investigations and opportunities for direct observation that complement textbook learning. Access to remote labs can also bring such experiences into the school classroom. For example, students can use a high-quality, distant telescope to make observations of the night sky during daytime school science classes.

Embodied learning Embodied learning involves self-awareness of the body interacting with a real or simulated world to support the learning process. When learning a new sport, 
physical movement is an obvious part of the learning process. In embodied learning, the aim is that mind and body work together so that physical feedback and actions reinforce the learning process. Technology to aid this includes wearable sensors that gather personal physical and biological data, visual systems that track movement, and mobile devices that respond to actions such as tilting and motion. This approach can be applied to the exploration of aspects of physical sciences such as friction, acceleration, and force, or to investigate simulated situations such as the structure of molecules. For more general learning, the process of physical action provides a way to engage learners in feeling as they learn. Being more aware of how one's body interacts with the world can also support the development of a mindful approach to learning and well-being.

Adaptive teaching All learners are different. However, most educational presentations and materials are the same for all. This creates a learning problem, by putting a burden on the learner to figure out how to engage with the content. It means that some learners will be bored, others will be lost, and very few are likely to discover paths through the content that result in optimal learning. Adaptive teaching offers a solution to this problem. It uses data about a learner's previous and current learning to create a personalised path through educational content. Adaptive teaching systems recommend the best places to start new content and when to review old content. They also provide various tools for monitoring one's progress. They build on longstanding learning practices, such as textbook reading, and add a layer of computerguided support. Data such as time spent reading and self-assessment scores can form a basis for guiding each learner through educational materials. Adaptive teaching can either be applied to classroom activities or in online environments where learners control their own pace of study.

Analytics of emotions Automated methods of eye tracking and facial recognition can analyse how students learn, then respond differently to their emotional and cognitive states. Typical cognitive aspects of learning include whether students have answered a question and how they explain their knowledge. Non-cognitive aspects include whether a student is frustrated, confused, or distracted. More generally, students have mindsets (such as seeing their brain as fixed or malleable), strategies (such as reflecting on learning, seeking help and planning how to learn), and qualities of engagement (such as tenacity) which deeply affect how they learn. For classroom teaching, a promising approach is to combine computer-based systems for cognitive tutoring with the expertise of human teachers in responding to students' emotions and dispositions, so that teaching can become more responsive to the whole learner.

Stealth assessment The automatic data collection that goes on in the background when students work with rich digital environments can be applied to unobtrusive, 'stealth', assessment of their learning processes. Stealth assessment borrows techniques from online role-playing games such as World of Warcraft, in which the system continually collects data about players' actions, making inferences about their goals and strategies in order to present appropriate new challenges. This idea of embedding assessment into a simulated learning environment is now being extended to schools, in topics such as science and history, as well as to adult education. The claim is that stealth assessment can test hard-to-measure aspects of learning such as perseverance, creativity, and strategic thinking. It can also collect information about students' learning states and processes without asking them to stop and take an examination. In principle, stealth assessment techniques could provide teachers with continual data on how each learner is progressing. However, much research remains to be done, both to identify the measures of student learning process that predict learning outcomes for different learning systems and to understand the amount and format of student learning data that are useful to teachers. Concerns have been raised about collection of vast amounts of student learning data and the ethics of using computers to monitor a person's every action. 


\title{
Introduction
}

\author{
This is the fourth in a series of annual reports on innovations in teaching, \\ learning and assessment. The Innovating Pedagogy reports are intended \\ for teachers, policy makers, academics and anyone interested in how \\ education may change over the next ten years.
}

This report is the result of collaboration between researchers at the Institute of Educational Technology in The Open University and the Center for Technology in Learning at SRI International. We have shared ideas, proposed innovations, read research papers and blogs, and commented on each other's draft contributions. We compiled the report by first producing a long list of new educational terms, theories, and practices, then reducing these to ones that have the potential to provoke major shifts in educational practice. This 2015 report introduces ten pedagogies that either already influence educational practice or offer opportunities for the future. By 'innovative pedagogies' we mean theories and practices of teaching, learning and assessment for the modern, technology-enabled world.

\section{4f six overarching themes: scale, connectivity, reflection, extension, embodiment, and personalisation $\boldsymbol{y}$}

We are aware that innovative pedagogies are proliferating like fundamental particles in physics. What started as a small set of basic teaching methods (instruction, discovery, inquiry) has been extended to become a profusion of pedagogies and their interactions. So, to try to restore some order, we have examined the previous reports and identified six overarching themes: scale, connectivity, reflection, extension, embodiment, and personalisation. In describing these, we have highlighted in bold the pedagogies identified in our previous reports.

\section{Scale}

Delivering education at massive scale has been the headline innovation of the past three years. Massive Open Online Courses (MOOCs) now engage millions of people in learning online. It is not clear whether the business of offering university-level courses for free can be sustained. However, MOOCs have demonstrated that it is possible to design methods of learning that improve with scale. Where the pedagogy of a MOOC is based on learning through conversation and social networking, then the more people who take part, the richer the interactions, with people around the world exchanging ideas and sharing perspectives. In July 2015, the largestever gathering of learners took place on the FutureLearn platform, with 270,000 people taking a course from the British Council on preparing for the IELTS language examination. Just one video from that course, asking the participants to discuss how they feel about taking exams, attracted 56,000 comments and responses. To manage this level of engagement requires techniques from social networks, of 'liking' comments, 'following' learners and educators, and rewarding popular learners and their contributions, so the most successful contributions are highlighted.

Other pedagogies that are being explored at massive scale include badges to accredit learning, crowd learning (participants post questions, stories, images, videos and computer programs for other learners to answer or review), citizen inquiry (members of the public propose and engage in investigations and science projects) and rhizomatic learning (learners work together in dynamic ways to determine their own curriculum and modes of learning). 


\begin{tabular}{|c|c|}
\hline Themes & Pedagogies (with year of report) \\
\hline \multirow[t]{6}{*}{ Scale } & Rhizomatic learning (2012) \\
\hline & MOOCs $(2012,2013)$ \\
\hline & Crowd learning (2013) \\
\hline & Citizen inquiry (2013) \\
\hline & Badges to accredit learning (2013) \\
\hline & Massive open social learning (2014) \\
\hline \multirow{4}{*}{ Connectivity } & Seamless learning $(2012,2013)$ \\
\hline & Flipped classroom (2014) \\
\hline & Bring your own devices (2014) \\
\hline & Crossover learning (2015) \\
\hline \multirow{5}{*}{ Reflection } & Assessment for learning (2012) \\
\hline & Learning analytics $(2012,2013)$ \\
\hline & Learning to learn (2014) \\
\hline & Learning design informed by analytics (2014) \\
\hline & Learning through argumentation (2015) \\
\hline \multirow[t]{9}{*}{ Extension } & Geo-learning (2013) \\
\hline & Learning from gaming (2013) \\
\hline & Event-based learning (2014) \\
\hline & Learning through storytelling (2014) \\
\hline & Threshold concepts (2014) \\
\hline & Computational thinking (2015) \\
\hline & Context-based learning (2015) \\
\hline & Incidental learning (2015) \\
\hline & Learning by doing real science (2015) \\
\hline \multirow{3}{*}{ Embodiment } & Maker culture (2013) \\
\hline & Bricolage (2014) \\
\hline & Embodied learning (2015) \\
\hline \multirow[t]{5}{*}{ Personalisation } & Personal inquiry learning (2012) \\
\hline & Dynamic assessment (2014) \\
\hline & Adaptive teaching (2015) \\
\hline & Analytics of emotions (2015) \\
\hline & Stealth assessment (2015) \\
\hline
\end{tabular}

Pedagogy themes that have emerged from the Innovating Pedagogy reports 


\section{Connectivity}

Learning at scale offers opportunities for connectivity between learners from different nations, cultures and perspectives. The opportunity now is to understand how to create and manage courses that encourage productive discussion on controversial topics.

Connectivity not only covers learning between people, but also across locations. Flipped classrooms allow students taking courses in conventional schools and universities to learn the basic principles of a subject by watching videos and reading instructional text online, then meeting with tutors on campus to explore and discuss the topic in greater depth. In crossover learning, students may start an investigation in class, initiated by a teacher, then continue it outdoors or at home, using mobile devices such as smartphones to collect data and evidence that are then shared and presented back in class. These are specific kinds of seamless learning, connecting learning experiences across locations, times, devices and social settings. A policy of bring your own devices not only supports these teacher-managed forms of connected learning, but also allows students to follow their own lines of research and collaborative learning inside and beyond the classroom.

\section{Reflection}

All this activity online and in the physical world could suggest a future for education that is hugely dynamic and mobile. That may be one vision. But knowledge also comes from reflection and contemplation. The engine of learning is a continuous cycle of engagement and reflection, with our activity in the world - as we explore an environment, perform an experiment, or read a book - producing new information that must be assimilated with existing knowledge. This provides both the enrichment and the mental conflict that are sources for reflection and understanding, perhaps leading to discussion and plans for further investigation. This cycle of productive learning appears in school classrooms (where teachers encourage reading, reflection and discussion), the science lab (through experiment, note taking and synthesis of data), and field or museum trips (where students form an inquiry question, collect data in the field, then reflect on the findings at home or in the classroom). By learning the skills of structured argumentation, students can explain their reflective processes to others, thus engaging in a collaborative process of experimentation and discussion. This is part of a process of learning to learn.

Assessment for learning can help each learner to reflect on current learning difficulties, find relevant resources and overcome difficulties. At its most effective, this kind of formative assessment fits into the cycle of learning, providing feedback on how well new information has been learned and giving pointers to new learning activities that will fill gaps in knowledge.

Learning analytics, which enable data on processes and outcomes of learning to be used to improve the quality of teaching, offer a means for educators to reflect on how they teach and on the ways in which they design for learning. More broadly, schools and universities can introduce a process of institutional learning, with everyone, including students, using idea-sharing software such as IdeaScale to reflect on successes and failures, and propose ideas to improve the quality of education.

\section{Extension}

Some innovative pedagogies form part of this process of institutional improvement not by offering radically different ways of teaching, but by extending the scope of current teaching methods and overcoming their weaknesses. Threshold concepts are ideas that open up new ways of thinking about a problem. If a threshold concept such as 'heat transfer' or 'centre of gravity' is taught well, it can inform everyday activities such as cooking or sports coaching.

Learning through storytelling offers new perspectives on an ancient tradition through techniques such as practomime that blend classroom and online storytelling, with teachers and students creating shared stories. Computational thinking offers a powerful approach to solving problems using structured techniques derived from computing, including iteration, debugging and problem decomposition.

Technologies also enable us to extend the settings in which learning takes place. Students can now learn by doing real science, making use of lab equipment and expensive technology that is not 
available on site but can be controlled remotely at distant locations. A variety of technologies, from televisions to augmented reality, also provide opportunities for event-based learning and context-based learning.

Management and orchestration of learning are essential to ensure that students are learning productively. As educators, researchers, and policy makers, we need to move beyond assumptions that playing games and chatting online with friends are necessarily bad. Instead, we should look for ways to integrate the worlds of social media, gaming and formal education. This will not be easy, given entrenched views, and it cannot be done in a naïve way, by adding a layer of game playing or social chat to traditional schooling. Perhaps the greatest challenge, and opportunity, facing education is to connect the productive incidental learning - that goes on in homes, workplaces, museums and the outdoors - with formal classroom education. This connection could be made using learning from gaming (an approach that enables employees to gain occupational skills of decision-making, strategy and negotiation through playing online strategy games) or geo-learning (students explore their local environment with the support of interactive maps and guides while connecting with other learners investigating the same environment online).

\section{Embodiment}

School, university and online learning all promote the primacy of abstract academic knowledge. Yet embodied learning recognises that we are creatures with bodies that we use to explore, create, craft, and construct. A renewed interest in maker culture has seen people gathering for maker faires, jamborees and craft days.

Enthusiasts use modern tools, such as Raspberry Pi hobbyist computers or 3D printers, to carry out environmental surveys, create soccer-playing robots, or design intricate jewellery. Bricolage is a practical process of learning by tinkering with materials, transforming products or materials that are ready to hand into new constructions. It is a fundamental process of playful learning, from building sandcastles to creating improvised art and fashionable clothing.

\section{Personalisation}

Continuing the physics analogy, personalisation is the mysterious missing particle of education. Since the early experiments with teaching machines in the 1950s, educational technologists have attempted to develop new methods of personalised instruction that will respond to the behaviour of each student, or infer students' mental states and correct their misunderstandings. So far, this has only been successful for limited topics in mathematics or science.

Adaptive teaching now offers the promise of using data about each learner's previous and current learning to create a personalised path through educational content. At the same time, analytics of emotions promise to provide personalised learning based on emotional responses, and personal inquiry provides opportunities for investigations based on a learner's own questions and interests.

Personalised learning is a wider process of understanding and developing the aptitudes and skills of each learner through methods such as dynamic assessment and stealth assessment. Is personalisation incompatible with learning at scale? Or will we be able to develop new pedagogies that offer thousands of learners the opportunity to pursue their personal pathways to knowledge, at the same time as they engage in shared discussion and collaborative inquiry?

\section{Innovating Pedagogy}

In our reports, we have aimed to understand and acknowledge learning in a world of interactive digital technologies. A focus on technologies could run the risk of chasing each invention up and down the switchback of innovation, marketing, hype and obsolescence. Some devices for education that have long been out of fashion, such as teaching machines, language labs, and integrated learning systems, reappear in new guises for the next wave of technology. By examining innovative pedagogies, we aim to ride the roller coaster of technology adoption, highlighting ways of teaching, learning and assessing that can be successful both now and in the future. 


\section{Resources}

A provocative and enlightening blog post

dealing with the perils of predicting the future of technology for education:

followersoftheapocalyp.se/i-watch-the-ripples-

change-their-size-but-never-leave-the-stream-

altc-2015/

IdeaScale software to create and share ideas:

Ideascale.com 


\title{
Crossover learning
}

\author{
Connecting formal and informal learning
}

\author{
Potential impact: high \\ Timescale: medium ( $2-5$ years)
}

We spend our lives learning yet choose to label particular sections more formally using terms such as 'kindergarten', 'school', 'university', 'qualifying' or 'professional development'. Such distinctions are becoming less useful as formal aspects of learning are interlinked with the informal learning experiences that occur during museum visits, afterschool and hobby clubs, or internships. Connected technologies, new approaches to assessing and recognising learning, and new insights into the value of informal learning are combining to blur the familiar distinctions. This section looks at the ways we can connect formal and informal learning experiences, benefiting from the crossover between them.

As well as being valuable in their own right, informal learning experiences can also enhance students' academic pursuits. Learning outside school supports the development of skills and dispositions that help students do better within school. A simple example of crossover from formal to informal learning is the museum visit, an experience which has obvious educational aspects but which varies depending on its structure and purpose. For example, setting formal aims focused on gathering evidence linked to subject-based questions will guide the actions of student visitors and connect the visit to the curriculum.

On the other hand, creating space in the formal curriculum for students to pursue individual themes based on their interests can enable informal experiences to influence curriculum topics and tasks. Authentic experiences with a subject, for example through an internship or mentoring role, can also encourage persistence in the pursuit of related careers. A more coordinated approach involves organisations such as museums, youth and hobby clubs partnering with schools and universities to develop learning materials aligned to the local curriculum.
The concept of crossover learning can also be applied to the ways in which we think about learning as a whole and the scope for adjusting how formal and informal combine to influence attitudes and motivation for learning at all ages. Increasingly, educators, policymakers, and researchers view learning as taking place across settings and contexts, in a 'learning ecosystem'. This perspective has coincided with a rise of interest in, and opportunities for, crossover learning, helping learners to connect experiences gained throughout the learning ecosystem. Research on linking informal and formal learning therefore covers aspects that help us consider learning in different ways, such as learning design, activity design, rethinking assessment and recognition, and the ways in which methods and technology can help to transfer information and experience across settings.

\section{Towards competency-based learning}

Some schools and universities are planning to include informal and non-academic learning practices as they move away from education based on 'seat-time' and homework, towards a competency-based approach that focuses on the ability to achieve goals and acquire skills rather than on the volume of knowledge gained. Recognising that students' informal learning can contribute to their development of skills and competencies may reduce student workload and allow opportunities for undirected activities outside the classroom to become part of schoolwork.

At one extreme, incorporating informal learning within the classroom could simply lead to reduced expectations for formal outcomes. But activities can also be designed that enable learners to recognise and reflect on their out-of-school activities. Also, 


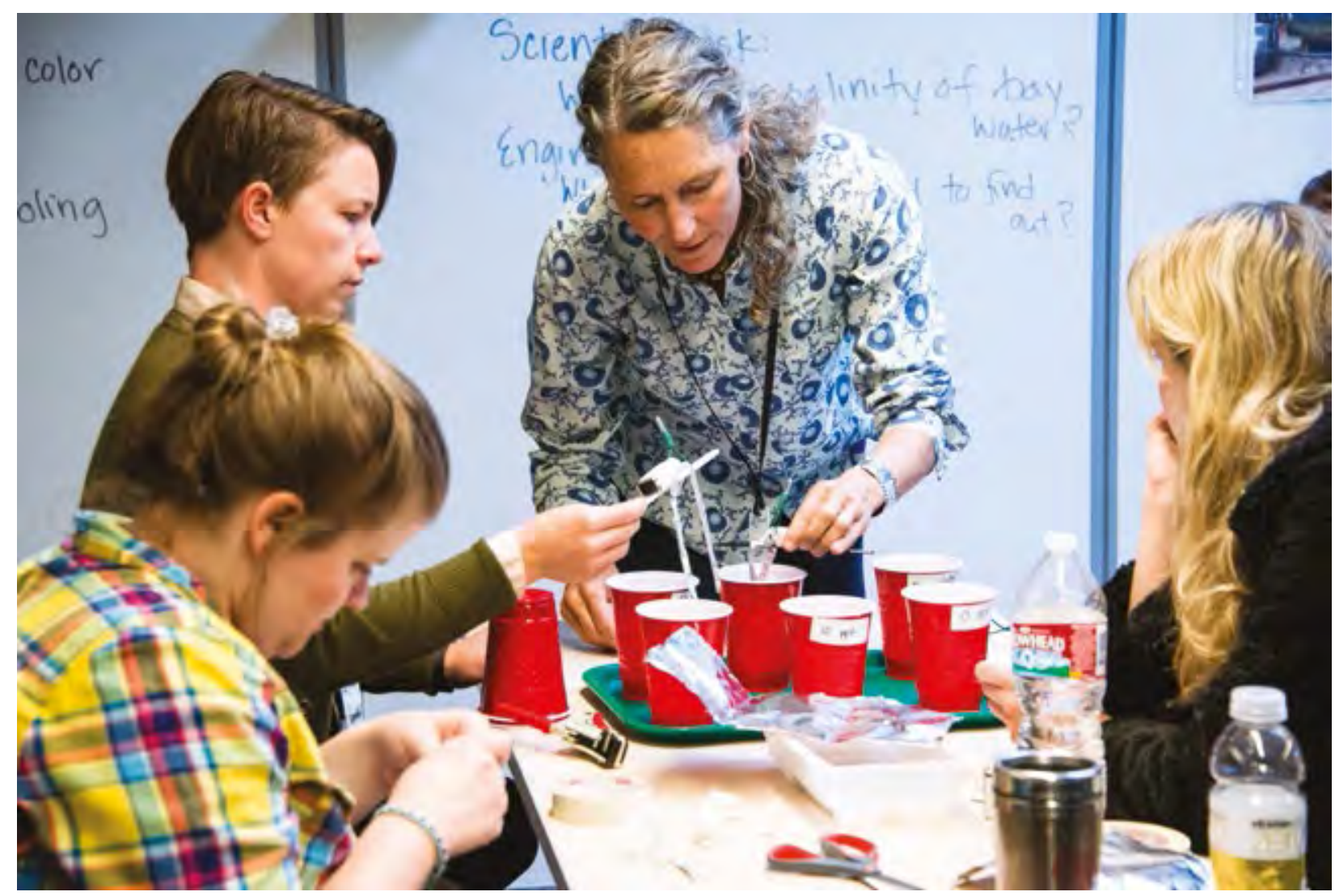

Session by the Teacher Institute at the Exploratorium, San Francisco, on 'Reengineering your Science Curriculum".

by engaging in practical activities, they can be supported to develop traits and skills such as persistence and self-direction that can aid success in any subject. For example, in an innovative project, students studying American Literature built their own chairs and, in the process, developed skills of problem solving, communication and collaboration. Identifying connections between the poetry they were studying and furniture making helped them appreciate the craft and structure of written texts.

\section{Recognising achievement}

A shift towards crossover learning requires adjustments to the ways in which we assess and recognise achievement. For example, using badges to record less formal achievements recognises activities that come from different sources. Tools that allow students to gather resources, such as Tumblr or Pinterest, can enable learners to develop transferable skills such as curation, evidence building and reflective commenting. Users of such tools provide records of their interactions and paths through information on the Internet, so that one item or a single collection can act as an entry point for a deeper exploration of a subject.
Teachers and informal educators now have more opportunities for professional development related to crossover learning. Those working in informal learning at museums and community centres can access the local curriculum and crossover key concepts and develop intentional connections between programme activities and school learning. Similarly, teachers can learn to facilitate open-ended explorations in their classrooms. The Teacher Institute at the Exploratorium, a museum that emphasises hands-on activities in San Francisco, California, helps teachers facilitate activities based on the principles behind the interactive exhibits at the museum. Partnerships between formal and informal education entities can help ensure that staff strategies for crossover learning are sustained.

\section{If Finding space to bring informal learning into formal education has the potential to enrich knowledge with פע experience}




\section{Conclusion}

Finding space to bring informal learning into formal education has the potential to enrich knowledge with experience. Adding direction to informal activities can enhance motivation and increase the impact of informal experiences on school learning and in the workplace. The challenge is to design this integration so that it retains the coherence of the established curriculum while embracing some of the fun and freedom of informal exploration.

\section{Resources}

Partnerships between art museums and schools: b l o g . e d . g o v / 2015 / 06 / 16 - m u s e u m s - i n partnership-with-schools-a-model-for-learning/

The Exploratorium Teacher Institute, San Francisco, USA:

exploratorium.edu/education/teacher-institute

Weidinger, N. (2012). Maker Education and The WikiSeat Project, The Institute for the Future Blog. iftf.org/future-now/article-detail/makereducation-and-the-wikiseat-project/

Example of crossover learning between an elementary school and local museums: jacksonville.com/news/metro/2015-05-10/story/ museum-magnet-creates-little-curators-anddocents-cross-over-learning
Report exploring the relationships between science education in formal and informal settings: Bevan, B., Dillon, J., Hein, G.E., Macdonald, M., Michalchik, V., Miller, D., et al. (2010). Making science matter: collaborations between informal science education organizations and schools. Washington DC: Center for Advancement of Informal Science Education. www.informalscience.org/documents/ MakingScienceMatter.pdf

National Research Council. (2015). Identifying and supporting productive STEM programs in out-of-school settings. Washington, DC: National Academies Press.

www.nap.edu/catalog/21740/identifying-andsupporting-productive-stem-programs-in-outof-school-settings 


\title{
Learning through argumentation
}

\author{
Developing skills of scientific argumentation
}

Potential impact: medium

Timescale: short (1-2 years)

Modern education extends beyond the transmission of knowledge and procedures, towards enabling students to become active and reflective learners. The pedagogy of argumentation prepares students for a world where the consequences of science, such as climate change and genetic engineering, affect every person and are publicly debated.

Students can only understand scientific ideas in depth by engaging in the kinds of inquiry and communication processes that scientists use. These involve reasoning and arguing from available evidence in order to improve and refute ideas and explanations, while communicating understanding through the use of precise language. The methods of scientific argumentation are not confined to the traditional sciences, but can be applied to mathematics, history, language, arts, and the human sciences. Science content and the practices of science need to be learned in concert; neither should be taught in isolation or as a prerequisite for the other.

\section{\&Argumentation pedagogy invites students to make claims and provide} supporting evidence $y$

Engaging students in scientific argumentation contrasts with traditional science pedagogy based on lecturing and posing closed questions to which the answers are already known. Argumentation pedagogy invites students to make claims and provide supporting evidence for those claims and to discuss whether the evidence someone presents for a claim is sufficient and is justified according to the standards of the discipline.

\section{How teachers can encourage productive argumentation}

To benefit from argumentation, students must listen and talk carefully, justify claims, and discuss ideas using reason and evidence. This kind of classroom discussion does not come easily to most students and needs to be thoughtfully supported. Teacher practices associated with learning through argumentation include:

- having students articulate their ideas orally and in writing

- asking questions that drive students to evaluate and improve their ideas

- restating or revoicing students' remarks in more scientific or mathematical language

- having students develop and use models to construct explanations.

Professional development can help teachers to lead dynamic group discussions using these strategies. Teachers can support constructive argumentation by establishing classroom norms for taking turns in conversation, active listening, and responding constructively to other people's ideas.

\section{Technologies to support learning through argumentation}

A good way to spark serious discussion of scientific ideas is to pose a thought-provoking question that has no simple answer and that requires discussion of theory and evidence. Here are some examples, ranging across topics and levels. Why aren't birds electrocuted when they land on electric cables? Why don't we feel the weight of a plane when it flies over us? Can we measure intelligence? Is time travel possible? How can we know whether Jesus was a real person? 
Teachers may ask students to investigate a topic in groups and then share and compare their responses. Classroom communication technologies can help this process. Classroom 'clickers' are devices handed out to each student in a class to indicate a response to a question. A teacher might ask students to suggest different answers to a question and then vote for the best answer, displaying a bar chart of the number of students selecting each response. The teacher on middle and secondary school topics in biology, chemistry, earth science, and physics. These projects present a driving question and then lead students through a process of online investigation that requires them to formulate arguments and explanations backed with data collected in the course of the investigation. Many of the projects lead to a student debate that pits different proposed solutions or conclusions against each other with the goal of further refining students' thinking.

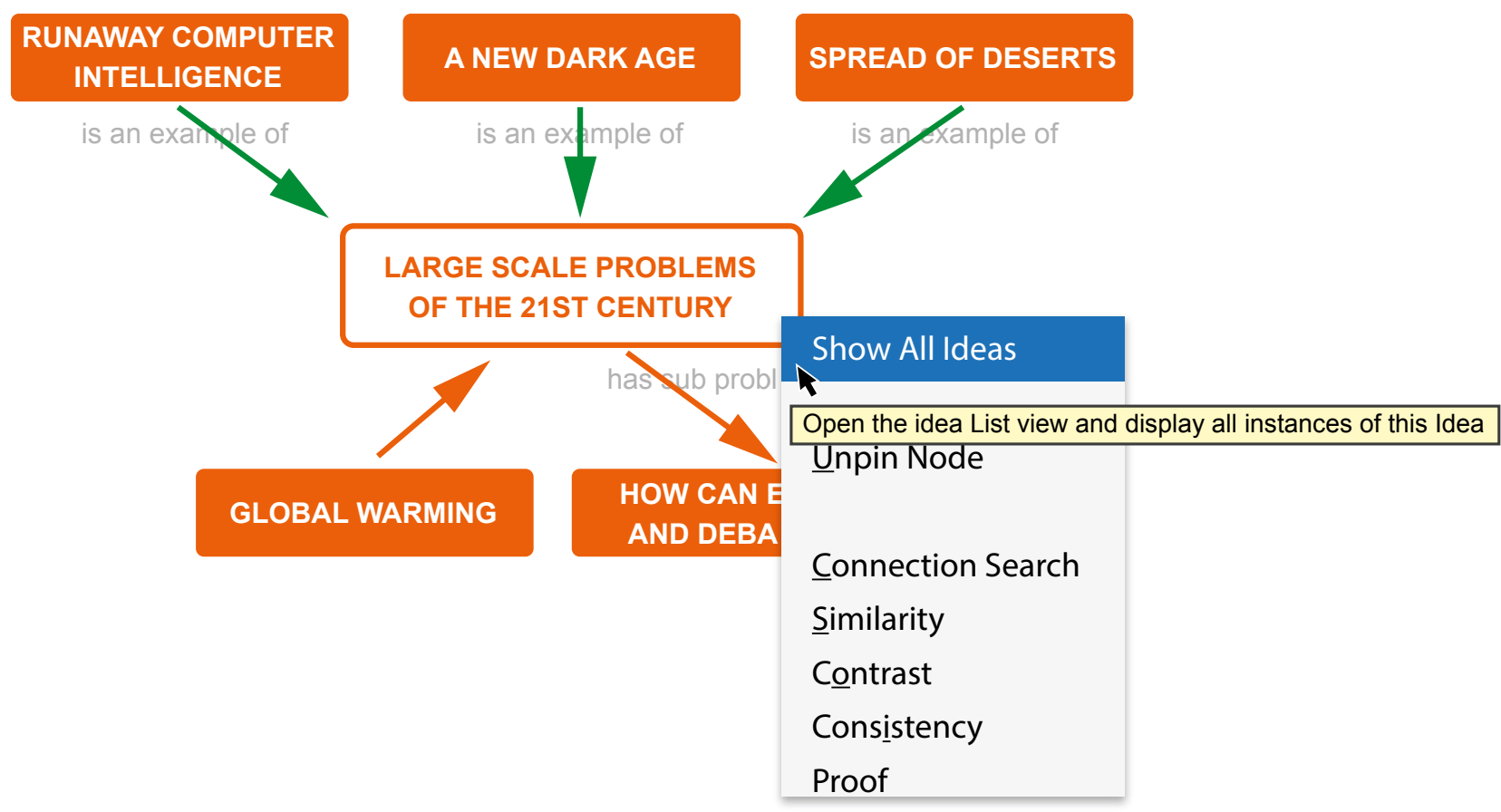

Argument visualisation in Cohere

then asks the class to discuss the responses, perhaps adding further evidence, then they vote again. Typically, student responses start to converge towards a more normative understanding of the scientific topic.

Students can also engage in scientific argumentation online. For example, the Cohere system supports visualisation of arguments. The Knowledge Forum online environment supports students to articulate, link, and reflect on their own and each other's ideas. Students have used Knowledge Forum to build an understanding of scientific topics such as human body systems and causes of pollution.

The Web-based Inquiry Science Environment (WISE) platform offers a variety of science projects

\section{Conclusion}

Implementing argumentation pedagogy can be difficult. Most students and teachers are used to questions with known answers, which are asked so students can demonstrate individual mastery of a science idea or topic. By contrast, argumentation provides an opportunity for students to build knowledge through a process of proposing, critiquing, and defending ideas over cycles of turn-taking. This can be a laborious process. Students need thoughtful teacher-guided support to learn the specialised form of argumentation that scientists use to build deeper understanding of the natural world. Teachers may require several years to become proficient in leading classroom discussions that promote the development of 
science knowledge and argumentation skills. Developing rich, curriculum-aligned questions or topics for science argumentation can be difficult as well.

Fortunately, freely available online resources provide examples that have worked well for teachers in the past. Well-designed science learning activities that incorporate rich topics for discussion in person or online can be combined with technologies to support communication and maintain a record of student thinking and its evolution over time.

\section{Resources}

Knowledge forum online environment for knowledge building:

www.knowledgeforum.com/

20 big questions in science:

www. theguardian.com/science/2013/sep/01/20big-questions-in-science

Alozie, N. M., Moje, E.B., \& Krajcik, J.S. (2010). An analysis of the supports and constraints for scientific discussion in high school project-based science. Science Education, 94(3), 395-427. deeplolue.lib.umich.edu/bitstream/ handle/2027.42/69162/20365_ftp. pdf?sequence $=1$

Description of Cohere system for argument visualisation:

Buckingham Shum, S. (2008). Cohere: Towards Web 2.0 Argumentation. In Proceedings of COMMA'08: 2nd International Conference on Computational Models of Argument, 28-30 May 2008, Toulouse, France. IOS Press, 97-108. oro.open.ac.uk/10421/

Comprehensive survey of research into using 'clicker' technology in large classrooms: Caldwell, J.E. (2007). Clickers in the large classroom: current research and best-practice tips. CBE-Life Sciences Education, 6(1), 9-20. www.lifescied.org/content/6/1/9. full
Study of classroom conditions that promote, nurture and sustain argumentation practices among students:

Duschl, R. A., \& Osborne, J. (2002). Supporting and promoting argumentation discourse in science education. Studies in Science Education, 38(1), 39-72.

cset.stanford.edu/sites/default/files/files/ documents/publications/Osborne-Supporting and promoting argumentation discourse in science education .pdf

To provoke argument among educators, see: Kirschner, P. A. \& van Merriënboer, J. J. G. (2013). Do learners really know best? Urban legends in education, Educational Psychologist, 48(3), 169-183.

ocw.metu.edu.tr/pluginfile.php/3298/course/ section/1174/Do Learners Really Know Best. pdf

Practical guidelines for teachers to manage productive talk in science classrooms:

Michaels, S. \& O'Connor, C. (2012). Talk Science Primer. Cambridge, MA: TERC.

inquiry project.terc.edu/shared/pd/

TalkScience_Primer.pdf

Resnick, L. B., Michaels, S., \& O'Connor, M.C. (2010). How (well-structured) talk builds the mind. In D. D. Preiss \& R. J. Sternberg (Eds.), Innovations in Educational Psychology: Perspectives on Learning, Teaching, and Human Development. New York, NY: Springer. 163-194. www.Irdc.pitt.edu/bov/documents/resnick howwell-structuredtalkbuildsthemind_033012. pdf 


\title{
Incidental learning
}

\author{
Harnessing unplanned or unintentional learning
}

\author{
Potential impact: medium \\ Timescale: medium (2-5 years)
}

Incidental learning is learning that is unplanned and may be unintentional. It can happen at any time in any place: at home, while working, or on the move. Incidental learning is not usually led by a tutor, neither does it follow a structured curriculum nor result in formal certification. It frequently occurs within activities that learners engage in by choice and forms part of everyday work and leisure.

\section{How researchers have explored incidental learning}

Young children engage in incidental learning as they develop abilities to speak, play with toys and interact with their family and friends. Through unstructured play, they can learn problem solving, language use, social, physical, and self-regulatory skills.

In a study of incidental learning of arithmetic, children were tested on their ability to do sums that require approximation ('more or less'). The study found that $65 \%$ of the children aged 5-6 could answer problems such as: "If you had 24 stickers and I gave you 27 more, would you have more or less than 35 stickers?" It seems that many young children are able to do a form of approximate arithmetic without being taught, by incidental learning of the skills of estimation. These powerful and general techniques are not developed further when they start school and begin with $2+2=4$.

Schools are recognising that children can learn through play and discovery, making time for unstructured exploration. But there is still little awareness that young children starting school have already gained skills of estimation, creative problem solving, wordplay, and game design that could form the basis of a new kind of early years curriculum.
Incidental learning continues into adulthood, but is generally not valued by examiners or employers. Early research into incidental learning explored how people learned as part of their daily work routines through observation, conversation with colleagues, use of work tools, and problem solving.

\section{\&Schools are recognising that children can learn through play and discovery, making time for unstructured exploration $y$}

Researchers have identified factors that make incidental learning successful. These include: the goals of the learners, people nearby for discussion and interaction, the tools they have at their disposal, their location, and the time they have available. Awareness of these factors improves understanding of the incidental learning that takes place and enables the creation of environments that support incidental learning.

Persistence and confidence contribute to success in incidental learning. The social environment is also important since the incidental learning process often places learners in a vulnerable position because they have to ask for help. Incidental learners may follow a path similar to the inquiry learning approach: framing and refining questions, planning an investigative path, and identifying resources that may help. Or they may seek out situations where learning can occur, such as public seminars or book-reading clubs. 


\section{Assisting incidental learning}

Game designers embed opportunities for incidental learning within computer games by setting challenges and offering rewards, as well as by providing landscapes to be navigated, rules to be inferred, and the motives and actions of game characters to be interpreted. Some 'serious games' employ similar forms of incidental learning to teach language and cultural skills by immersing players in a foreign environment. This builds on the longstanding approach (from the European 'grand tours' of the 18th century) of taking young adults on trips abroad to pick up the language and culture of a foreign country.

Second language educators have long recognized the advantages of the context-richness and social learning experienced by incidental learners who are immersed in authentic foreign language environments. Travellers and migrants often pick up languages much faster than classroom students can. Techniques to emulate immersive incidental learning include encouraging learners to hold everyday conversations in the language they are learning, teaching exclusively in the language, and bringing examples of use (for example, those gained by watching a foreign movie with subtitles) back into the classroom for reflection and discussion.

Educators are also exploring how to design learning experiences that build upon incidental learning that takes place elsewhere. Approaches include support for learners in reflecting on learning that takes place throughout their lives and making connections between incidental learning and deliberate learning.

\section{New developments}

New developments have promise for extending the benefits of incidental learning. For example, the iSpot citizen science application is designed for people of all ages to spot and share their observations of wildlife. For example, a hiker can upload photos of an unusual bird, insect or flower to iSpot, adding basic information and, if possible, a suggested identification. Others respond by adding, amending or confirming the identification. Once confirmed, the software links this addition to other identifications of the species with photos by

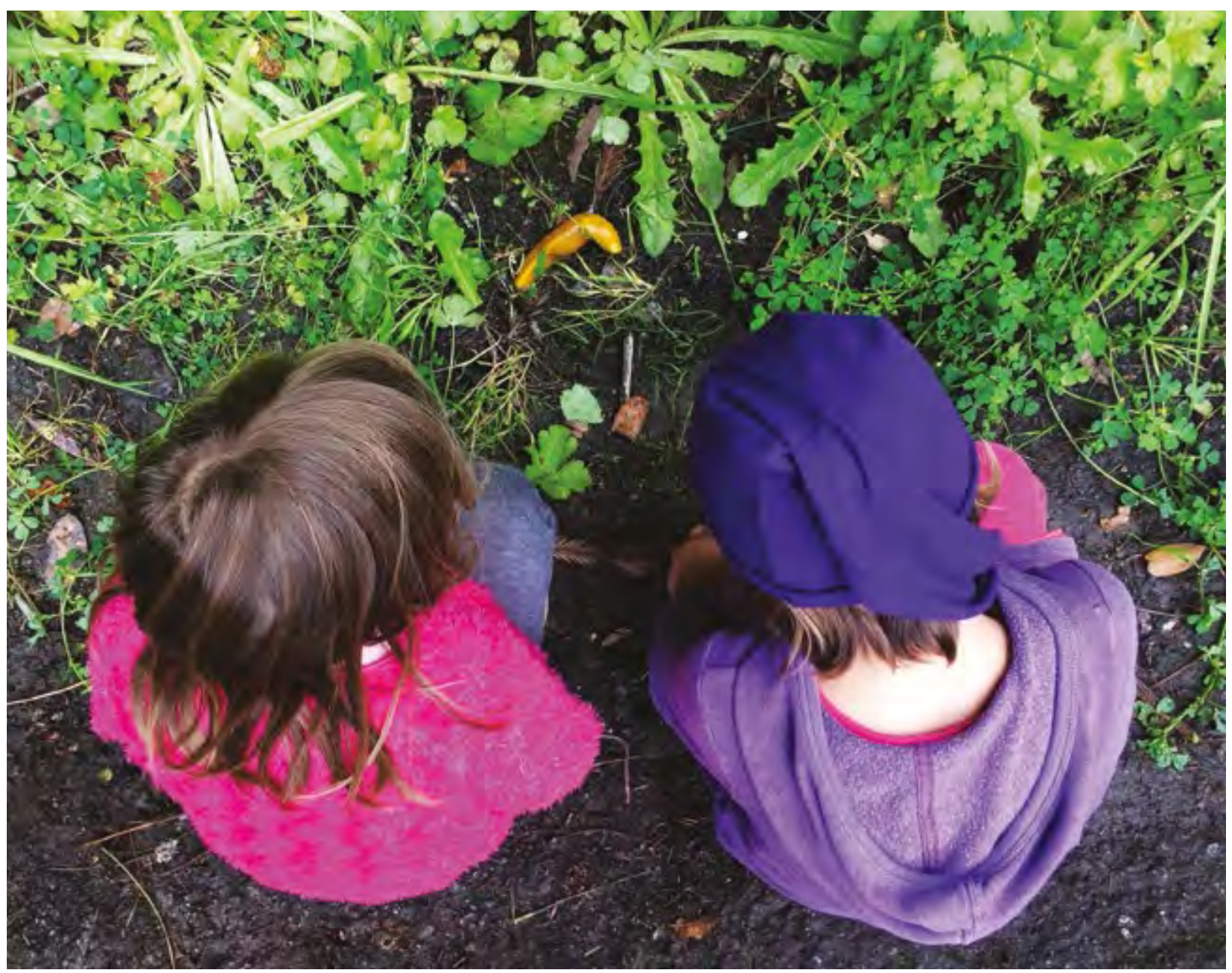

Children closely observe a banana slug during unstructured play 
their finders. This act of incidental learning can set the contributor on a path of discovery that includes comparisons with other sightings and a developing understanding of how experts classify species.

Based on principles of incidental work-based learning, SRI International is developing a mobile app to add a virtual mentoring structure to on-thejob learning. This includes ways for the employer and employees to generate useful content that may be shared by browsing a 'deck of cards' in the app. Learners can set goals based on available time and have progress tracked. They can also seek information on work topics and connect with colleagues engaged in similar tasks.

\section{Conclusions}

Although incidental learning can enrich formal learning and occurs throughout a lifetime, it presents challenges for teachers and learners. It can be difficult for a teacher to know when incidental learning has occurred, given that it cannot be planned and often is not recorded. There is also the challenge involved in providing learners with welltimed opportunities for reflection and helping them reconceive isolated learning fragments as part of more coherent, longer term learning journeys. Equally, there are challenges for learners to value their own learning journeys, find time to pursue their hobbies and interests, and resist attempts by others - parents, teachers, managers - to overformalise and validate their personal learning.

We still know very little about how young children acquire abilities related to language, arithmetic, science, and social interaction, let alone the beginnings of creativity, art appreciation, psychology and philosophy. As researchers start to uncover these processes of incidental learning, we may see the emergence of new pedagogies that build on children's pre-existing skills and develop these into adulthood.

\section{Resources}

iSpot community to identify wildlife

and share nature:

www.ispotnature.org/

Study of how pre-school children can solve addition and subtraction problems through approximate arithmetic:

Gilmore, C. K., McCarthy, S. E., \& Spelke, E. S. (2007). Symbolic arithmetic knowledge without instruction. Nature, 447(7144), 589-591.

www.psychology.nottingham.ac.uk/staff/lpzcg/ files/nature05850.pdf

Design of web-based systems to support incidental learning:

Glahn, C., Specht, M., \& Koper, R. (2009).

Perspective and Contrast, Design Principles for Supporting Self-directed and Incidental Learning. In Proceedings of I-KNOW' 09 and I-SEMANTICS '09, 2-4 September 2009, Graz, Austria. dspace.learningnetworks.org/ bitstream/1820/2009/1/perspective_and_ contrast_design_principles.pdf
Kerka, S. (2000). Incidental learning. Trends and Issues Alert No. 18: Center on Education and Training for Employment. www.calpro-online.com/eric/docs/tia00086.pdf

Le Clus, M. A. (2011). Informal learning in the workplace: a review of the literature.

Australian Journal of Adult Learning, 51(2), 355373.

ro.ecu.edu.au/cgi/viewcontent.cgi ?article=115 2\&context=ecuworks 2011

Silva, P. M. (2007). Epistemology of Incidental Learning. PhD, Virginia Polytechnic Institute and State University.

scholar.lib.vt.edu/theses/available/etd10162007-224008/unrestricted/silva.pdf

Application of psychological theory to overcoming the distinction between formal and informal education:

Strauss, C. (1984). Beyond 'formal' vs. 'informal' education: uses of psychological theory in anthropological research. Ethos, 12(3), 195-222. scholarship.claremont.edu/pitzer_fac_pub/66/ 


\title{
Context-based learning
}

\author{
How context shapes and is shaped by the process of learning
}

\author{
Potential impact: medium \\ Timescale: medium (2-5 years)
}

Context is how we make sense of experience, by distinguishing between what is relevant and irrelevant. For example, when reading a book, the meaning of each word and phrase is conveyed not only by its own characteristics, but also by its location in relation to other words or illustrations.

Until recently, education has been designed to minimise the effects of context on learning, so that children can gain universal knowledge and take tests that are appropriate whatever the location, time of day, or surroundings. Yet many professions, such as medicine, art, or engineering, require general professional knowledge to be applied in specific contexts. They also need practical knowledge to be built up from working in many differing situations. So an understanding of how context relates to learning is essential for innovating pedagogy.

\section{Learning in context and learning by creating context}

Consider a group of friends standing before a painting in an art gallery. They are in a specific context, comprising the painting, gallery, the friends and other people. They are also creating context by engaging in shared action and conversation, by moving closer to see the painting, discussing the artist, and comparing this painting with others they have seen. Thus context is both something we are immersed in and something we create. The same is true when reading a book: we are simultaneously at a specific word on a page, and creating contextual meaning from our knowledge of language and literature.

This dual nature of context, as something that surrounds us and something we create through our activity, raises problems for teachers at all levels. A teacher of young children needs to offer them opportunities to create context through exploratory play, while safeguarding them so they do not stray into dangerous situations either outdoors and online. At university level, a central issue in subjects such as geology, archaeology and environmental sciences, is whether to use a field trip to immerse students in an authentic context, with all its risks and uncertainties, or to provide them with an experience similar to that of a field scientist by manufacturing or simulating typical data.

\section{ff context is both something we are immersed in and something we create $y$}

Augmented reality, virtual reality and environmental modelling can provide students with the experience of viewing and sampling real data, for example by viewing a virtual microscope showing pre-prepared images of rock that can be zoomed, scanned and viewed under different lighting conditions. These technology-enhanced approaches increase ease of access and teacher control of the situation, but these advantages must be weighed against the value of doing science in real locations which are potentially difficult and costly to access.

\section{Context-sensitive technologies for learning}

New context-sensitive technologies offer opportunities to develop enriched contexts for learning. Handheld location-aware guides and augmented-reality applications can offer audio, text and images to describe the current location or object, such as a painting or museum item. The aim is to give the visitor general information that relates to the specific place or exhibit. 
The Aris application offers a set of tools to create and deliver location-based games such as scavenger hunts and re-enactments of historical events. One Aris game re-creates a student protest in the 1960s on the University of Wisconsin Madison campus. A visitor to the university can take the role of a reporter, moving round the campus, witnessing past events through images and video at the campus locations where the events took place and conducting simulated interviews with participants.

Users of Aris can also create contexts by taking photos and making notes that are added to a map,
Generalised knowledge about migration and climate change develops by exploring patterns of data across time and location.

\section{Context in education}

Young children's learning is tied to contexts of time, place, people, objects, so they live 'in the now'. As they mature, children are increasingly able to create context by applying generalised knowledge to a situation. For both children and adults, this process can be supported by tools for accessing knowledge in context and for abstracting general knowledge across multiple contexts.

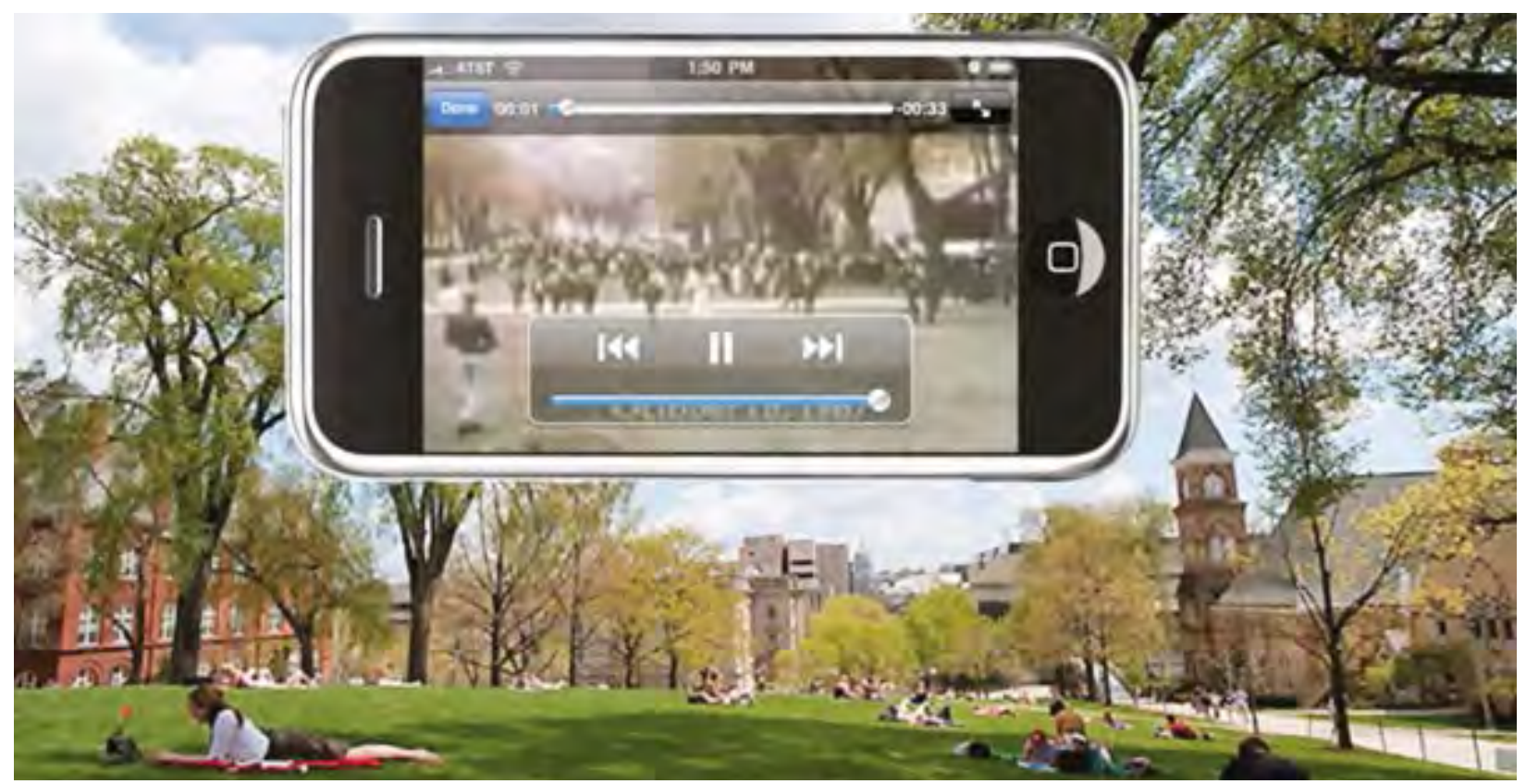

Aris context-based game showing the campus location of a student protest in the 1960 s

for others to view when they reach the appropriate locations. This and similar context-based systems can be used, for example, in citizen journalism, as people record everyday events, or in language learning for people to add labels in a foreign language to locations and objects that pop up on other people's devices when they visit each location.

When learning across contexts, communities of amateur scientists already share and compare local data on, for example, weather, wildlife, rocks and fossils. Schools can participate in these community activities through sites such as Journey North, which engages students and citizen scientists around the world in studying wildlife migration and seasonal change, including tracking migration of hummingbirds, recording sightings of butterflies and noting when tulips bloom in spring.
Contextual learning ties together pedagogies covered in previous editions of the Innovating Pedagogy report, including geo-learning, seamless learning, event-based learning, crowd learning, and citizen inquiry. The common theme is learning that comes from being situated in and understanding a context, and from reporting and comparing events across multiple contexts. As an approach to education, contextual learning involves helping students to learn from the world around them, and to see how general principles in science and society relate to their everyday lives.

It is often not easy to take what is learned in one setting and apply it in another. Words and ideas vary according to their context. A word like 'set' in English has very different meanings in a kitchen, a tennis court, and a mathematics classroom. Similarly, ideas and activities may need to be reinterpreted from one context to another, and data 
items collected at particular locations may need to be checked or modified before they can be used more generally. So context-based learning is a powerful means to understand places and events, and to connect general knowledge with everyday life, but it requires skill in interpreting localised words, concepts and data.

\section{Resources}

Aris web-based system for creating and playing context-based mobile games:

arisgames.org/

Luckin's Ecology of Resources design framework for learning, context and technology:

eorframework. pbworks.com/w/page/3777765/

FrontPage

Journey North website that engages students and citizen scientists in tracking wildlife migration and seasonal change:

www.learner.org/jnorth/
Discussion of the 'Salters' courses for context-based science education, focusing on development of the Salters Advanced Chemistry course:

Bennett, J., \& Lubben, F. (2006). Context-based chemistry: the Salters approach. International Journal of Science Education, 28(9), 999-1015. hal.archives-ouvertes.fr/hal-00513319/ document

Hansman, C.A. (2001). Context-based adult learning. New Directions for Adult and Continuing Education, 2001(89), 43-52.

www.andrews.edu/sed/leadership_dept/ documents/context_based_adult_.pdf 


\title{
Computational thinking
}

\author{
Solving problems using techniques from computing
}

\author{
Potential impact: medium \\ Timescale: medium (2-5 years)
}

As we learn chemistry, we broaden and extend our knowledge of the scientific method. As we study music, we develop and refine our sense of timing and of rhythm. Each area of the curriculum is associated with a set of skills that can be applied throughout our life, giving us new ways to understand the world.

In the case of computing, as we learn its principles and languages, we also acquire a set of problemsolving skills. Together, these are known as Computational Thinking. The value of these skills and their associated concepts, practices and perspectives, is widely recognised.

Countries are adding computational thinking to the list of key school subjects. In England, the National Curriculum states that children should be offered high quality computing education that will equip them with skills in computational thinking and creativity to understand and change the world. Outside education, large companies such as Google and Microsoft Research regard computational thinking as an essential set of problem-solving skills and techniques for software engineers.

\section{Elements of computational thinking}

Computational thinking uses the same steps to deal with a variety of problems.

Decomposition - breaking a large problem down into smaller ones

Pattern recognition - recognising how these smaller problems relate to ones that have been solved in the past

Abstraction - identifying and setting aside unimportant details

Algorithm design - Identifying and refining the steps necessary to reach a solution
Debugging - Refining those steps.

A final step that is usually implied without being included in the main list is

Presenting a solution in a usable form.

This method of problem solving can be worked through as a series of explicit steps. In the process, it may be internalised so that we use it automatically when faced with a new problem.

'Computational' does not imply that humans are being taught to think like unimaginative machines that can only solve a problem when supplied with a set of instructions to follow. Computational thinking is a way in which humans can think when they are trying to solve problems. The approach can be set out as a set of clear steps, but separating and navigating these steps is a creative human activity.

Although this way of thinking was developed in the context of computer programming and computer science, it can be applied more widely. Some definitions specify that it is used for the development of problem-solving procedures that can be mechanically interpreted. This appears to be an unnecessary restriction. Computational thinking provides a way of formulating problems and their solutions. It enables people to deal confidently with complexity and with open-ended problems. It is essential when developing computer applications, but it is also valuable in any discipline.

\section{Problem-based learning}

In the classroom, computational thinking can be related to the use of problem-based learning. This pedagogy was developed in response to the needs of professional practice, as a way of ensuring that students are not only able to pass examinations but can also apply in practice the concepts that they have learned. 
In order for problem-based learning to be effective, teachers need to ensure that students are familiar with the relevant skills and concepts, and that they are guided as they apply a problem-solving strategy. This is likely to involve working together to:

1. Examine a case and clarify terms

2. Identify the problem

3. Analyse the problem

4. Draft an explanatory model

5. Establish learning goals

6. Work individually to collect additional information

7. Apply and discuss additional information

For example, medical students might be presented with a set of information about a patient. Having clarified the details, they would list key symptoms and possible causes, suggest what might be wrong, identify what they need to find out in order to check if they are correct, perform some tests and then use these additional data to make a firm diagnosis.

This is a systematic approach to problem solving, designed for the classroom. It focuses on a single problem and includes learning goals. It shifts from group work to individual study and back to a

\section{\&s Studying computing involves much more than learning to code $5 y$}

plenary session. Although the individual work may be extensive, this is essentially a time-bounded approach. In a real-world setting, the process would extend further, with solutions tested and refined over a long period. In problem-based learning, the process ends at Step 7, at which point an appropriate solution to the problem is likely to be identified in discussion with a teacher.

Computational thinking differs from problem-based learning in that it arises from a need to solve immediate practical problems rather than work through pre-prepared exercises. It breaks an initial problem down into smaller elements, then relates these to ones that have been solved in the past. It also assumes that solutions will be tested and refined until an acceptable one is reached. Thus, it can be more useful in practical settings than the problem-based learning approach, but may not be appropriate to solve human and social problems that cannot easily be decomposed into subproblems.

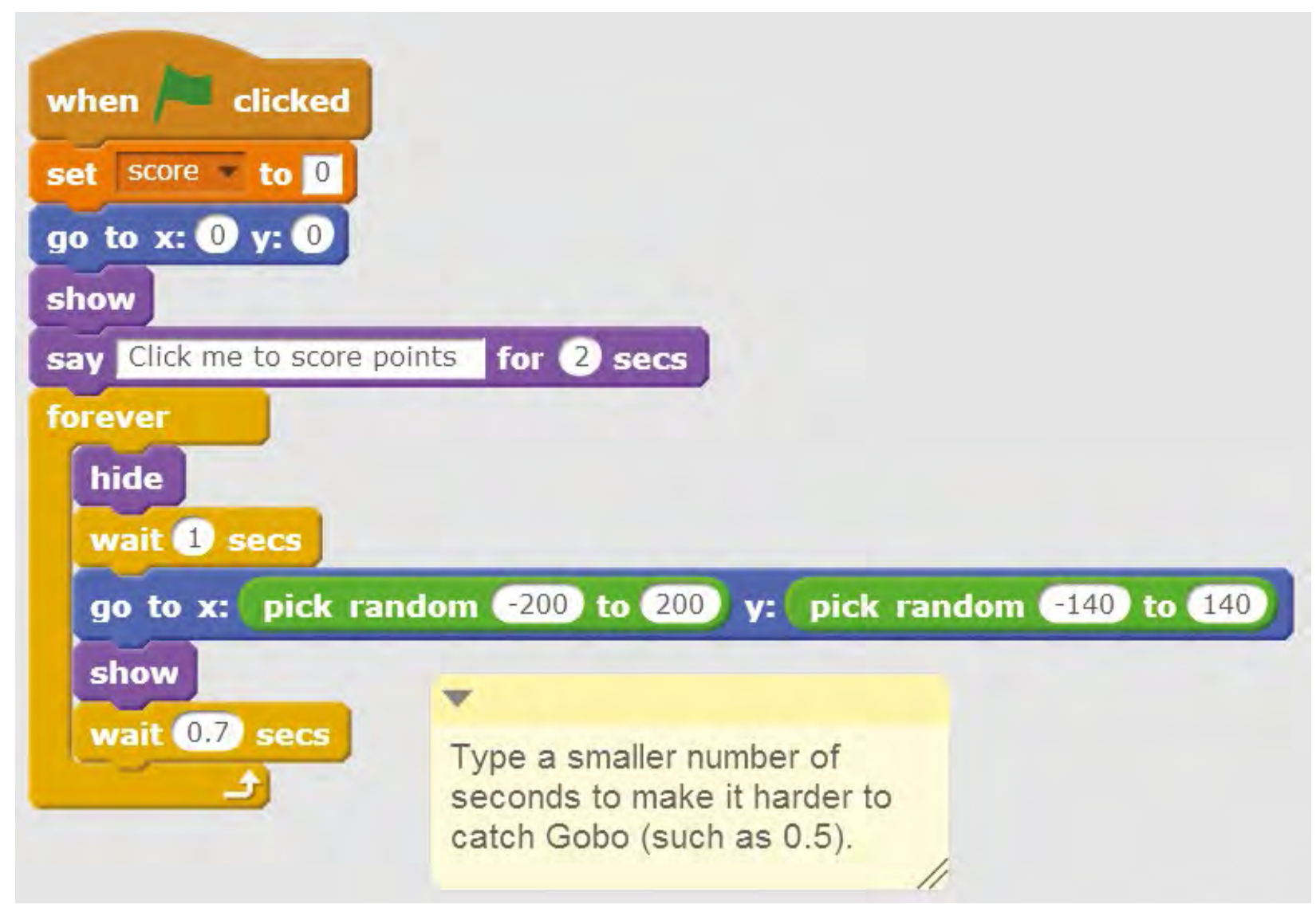

Section of Scratch code for a hide-and-seek game 


\section{Computational thinking skills}

Learning to solve problems in the context of computing is associated with the development of a set of skills. Researchers who have investigated how children engage with the programming environment Scratch have identified skills and shifts in perspective related to computational thinking:

Experimenting and iterating - developing something, trying it out, and then developing some more.

Testing and debugging - finding and solving problems as they work.

Reusing and remixing - building on existing projects or ideas

Abstracting and modularizing - exploring connections between the whole and the parts

Expressing - recognising that working in this way is a creative activity.
Connecting - recognizing the power of creating with and for others

Questioning - feeling empowered to ask questions about the world.

These findings emphasise that computational thinking is more than a set of steps to be worked through. It is not only a way of thinking, but also a way of working and of approaching problems.

\section{Conclusion}

Studying computing involves much more than learning to code. It enables learners to engage in abstraction, by defining patterns and generalizing from specific instances. It introduces ways of processing information and representing it in different ways. It requires learners to work systematically to identify and remove errors. Perhaps most importantly, it provides them with a way to break down problems and work to solve them across every area of life.

\section{Resources}

The importance of computational thinking and how it can be incorporated within the curriculum for children aged 5-11:

Berry, M. (2013). Computing in the National Curriculum: a guide for primary teachers. Bedford, UK: Computing at School. www.computingatschool.org.uk/dlata/uploads/ CASPrimaryComputing.pdf

Computational Thinking: Teacher Resources from The International Society for Technology in Education (ISTE) and the Computer Science Teachers Association (CSTA):

csta.acm.org/Curriculum/sub/CurrFiles/472.11

CTTeacherResources_2ed-SP-vF.pdf

Google for Educators offers a curated collection of resources related to computational thinking: www.google.com/edu/resources/programs/ exploring-computational-thinking/index. html\#!resources
What is Computational Thinking? A framework developed by Harvard, based on studies in the context of the Scratch programming environment: scratched.gse.harvard.edu/ct/defining.html

Scratch programming environment for children: scratch.mit.edu

A framework that includes lesson planning, classroom techniques and assessment methods:

Curzon, P., Dorling, M., Ng, T., Selby, C., \& Woollard, J. (2014). Developing computational thinking in the classroom: a framework. Computing at School. community.computingatschool.org.uk/ files/3517/original.pdf

A detailed review of publications on computational thinking:

Grover, S., \& Pea, R. (2013). Computational thinking in K-12: a review of the state of the field. Educational Researcher, 42(1), 38-43. 


\section{Learning by doing science with remote labs}

\section{Guided experiments on authentic scientific equipment}

\section{Potential impact: medium \\ Timescale: ongoing}

Laboratory experiences offer opportunities for students to experiment directly with the material world, or data from it, using the tools, data collection equipment, models and theories of science. That was the conclusion of a report from the US National Academy of Sciences, echoed in a statement from the British Council for Science and Technology that "Practical laboratory work is the essence of science and should be at the heart of science learning." Yet the value of school laboratory work has also been criticized, as the experience of running an experiment in a school lab can emphasize practicalities and neglect deep learning.

One important area of innovation has been to provide students in schools and universities with remote access to real scientific experiments. In a remote laboratory, a student controls real materials and apparatus over the internet, and the procedure can be executed flawlessly by a computer. Students can therefore focus on intellectual skills and conceptual understanding. Classroom teachers can spend less time on setting up and managing materials and equipment, and more time on framing and supporting student learning. Students can also more easily compare data sets, collect larger data sets, and engage in replications and extensions. An example is the Remote Experimentation Laboratory (RExLab) at the Federal University of Santa Catarina, Brazil. In Brazil, only $7 \%$ of schools currently have their own science labs, so it gives students the opportunity to control scientific equipment and run experiments in electrical circuits, mechanics, physics and computing.

Note that a remote laboratory is not a simulation: students control high-end scientific equipment and collect data from physical phenomena. Through a remote lab, students can now work with scientific apparatus and materials that would otherwise be too expensive, dangerous, difficult, or timeconsuming. For example, the Radioactivity iLab enables students to measure radiation from a sample of strontium-90. In this iLab, students in the United States move a Geiger counter in Australia up and down to measure the radioactivity at different distances and watch what happens over a live video. The learning goal is to observe and infer that the intensity of radiation from a point source decreases proportionally to the inverse square of the distance.

\section{Gf balance the time and support for doing the lab work with time and support for learning from it 5}

Remote labs are available for many topics including astronomy, biology, chemistry, computer networking, earth science, engineering, hydraulics, microelectronics, physics and robotics. Further, common platforms are emerging such as iLab Central, Go-Lab and the OpenScience Laboratory. The benefits and growing availability of remote labs mean that now is an ideal time to focus on the pedagogical innovations that will be necessary to realise the full potential of both local and remote labs.

As technology makes the mechanics of lab work faster and more straightforward, how can learning 
be enhanced? Six questions are at the heart of innovative pedagogies for learning by doing science:

What is the learning purpose? In traditional teaching labs, the purpose was sometimes only to handle scientific equipment safely and precisely. This work is taken out of students' hands in a remote lab. Consequently, in remote labs educators more often focus on conceptual understanding and inquiry meaningful experiments and investigations. They need help to form concepts, through techniques such as concept mapping, and to elaborate hypotheses. They also need considerable guidance in making sense of the data that results, relating the data back to their original driving questions, and deciding what to do next. By attending to the complete cycle of planning, acting and reflecting, educators can better develop students' abilities as selfregulating learners.

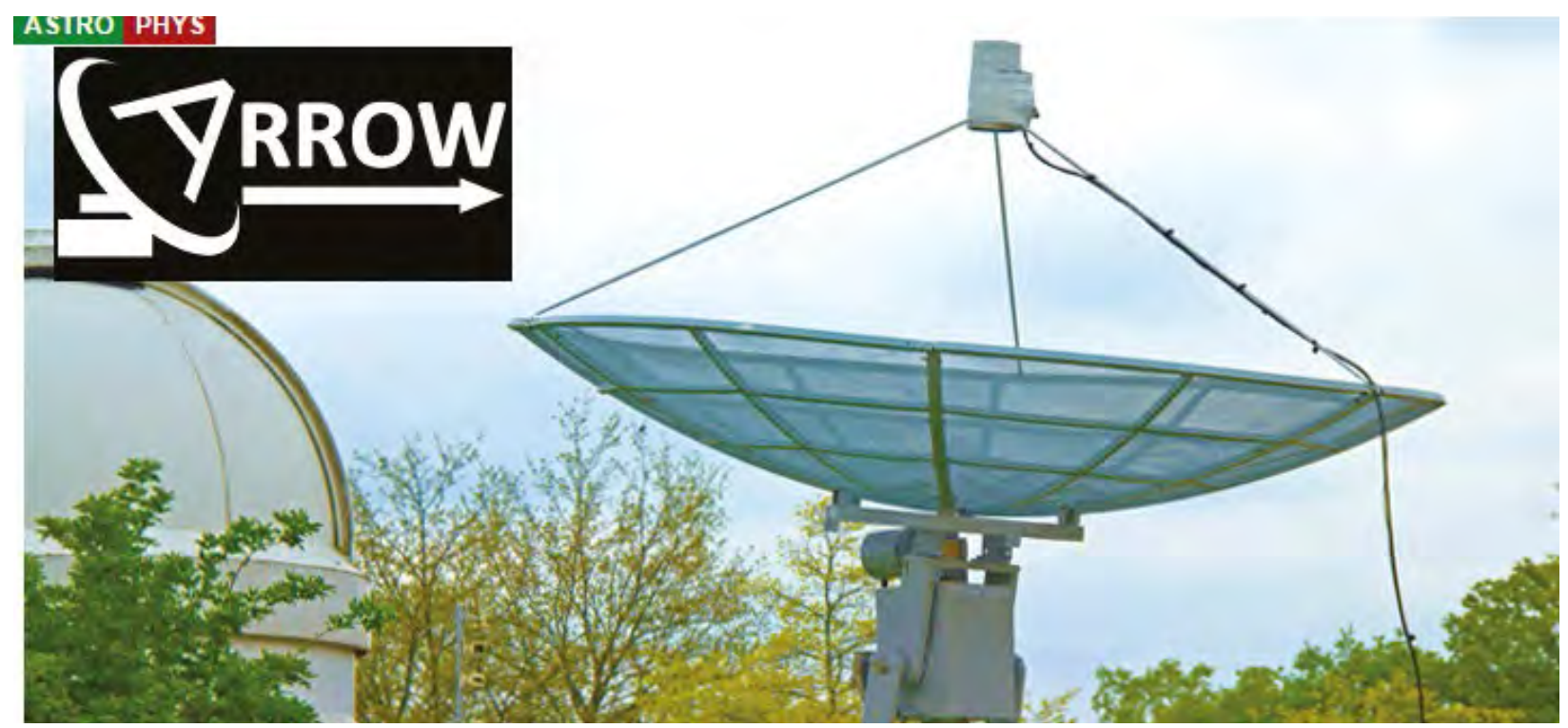

ARROW remotely controlled radio telescope for Open University students

goals that are aligned with today's curricular aspirations. Students often appreciate spending less time setting up (and cleaning up) a lab and more time pursuing intellectual goals. They may also appreciate getting a higher quality data set with less fine tuning of equipment. But, for some purposes, handson experience with local materials may be better. For example, in a remote lab students have fewer opportunities to design how they control and measure phenomena. Whether with remote or local labs, a focus on authentic science learning goals (and not just on the use of authentic materials) is a hallmark of innovative pedagogy.

What guidance do students need before and after the lab session? Educational designs often provide explicit resources for guiding students during the lab session. The guidance they require beforehand and afterwards is often less thoughtfully designed. For example, students need support to plan
How can students get timely feedback to guide their learning? Researchers have noted that, in traditional physical labs, teachers typically walk around to identify difficulties students are having with the lab, and intervene appropriately - however, they may need guidance on how to engage students conceptually during the session. In a remote lab, students also need to be tested and assisted during their engagement with the lab, but the teacher may not be present during the session. Digital resources can support students in checking their own understanding and progress, and thereby provide feedback to guide learning while doing the lab work.

How can social roles support learning goals? Likewise, physical labs in schools and universities are places where students interact socially in support of each other's learning. In remote labs, a basis for communication can be set up via chat or online calling. Yet communication alone is not enough. 
Research in the area of computer-supported collaborative learning emphasises the need to structure students' collaboration, which can include assigning specific roles to students, providing a shared workspace, orchestrating when students should communicate in the course of their work with the lab, and helping them to monitor and improve the state of their social engagement.

5 Can the places for sense making and data collection be flipped? Conventionally, students are expected to collect data during school time, and to organize and interpret their data as homework. However, this arrangement requires students to do challenging intellectual work in the less supported environment, alone at home. With remote labs it becomes possible for students to run their experiments outside class, thus flipping the use of time at home and in school. This could enable teachers to focus with students on making decisions about what to investigate and discussing the resulting data. Overall, innovative pedagogies can be employed to balance the time and support for doing the lab work with time and support for learning from it - making sure that the difficult work of sense-making is not left to students to struggle with alone.
How can teachers prepare? Remote labs offer new possibilities for teacher learning. For trainee teachers who are studying at university, they can practise with a remote lab on their university campus and then teach students with the same lab during a practical teaching experience in a school. Remote labs also offer teachers sample data sets that students collected, which can be used to help with planning their lessons. Further, since teachers in different locations can use the same remote lab, they can more easily engage in discussions of their pedagogical approach to teaching the lab class.

\section{Conclusion}

Doing real, practical scientific experiments is no longer restricted to science labs in schools or universities during normal working hours. The ability to conduct experiments remotely over the Internet means that students may have access to equipment and materials that they would not otherwise be able to engage with, due to issues around cost, safety, level of difficulty and time required. This also enables students and teachers to focus on learning goals and the pedagogy of science learning, rather than just practical handling of apparatus. 


\section{Resources}

Go-Lab portal to online labs for schools:

www.go-lab-project.eu

Open letter from the Council for Science and Technology to the former UK Secretary of State for Education in 2013, arguing that practical laboratory work should be at the heart of science learning:

www.gov.uk/government/uploads/system/ uploads/attachment_data/file/230509/13-1131stem-education.pdf

iLabs network of remote labs:

ilabcentral.org

Remote Experimentation Laboratory (RExLab) provided by the Federal University of Santa Catarina, Brazil:

relle.ufsc.br

OpenScience Laboratory, bringing practical science to students:

learn5.open.ac.uk

Review of remote labs in education:

Cooper, M. \& Ferreira, J. M. M. (2009). Remote laboratories extending access to science and engineering curricula. IEEE Transactions on Learning Technologies, 2(4), 342-353. oro.open.ac.uk/20433/1/tlt2009040342.pdf

Evaluation of use of remote labs with schools, indicating a need to focus on active manipulation of control devices and to teach key skills such as experimental design and control of variables: Lowe, D., Newcomb, P., and Stumpers, B. (2013). Evaluation of the use of remote laboratories for secondary school science education. Research in Science Education, 43(3), 1197-1219.

link.springer.com/article/10.1007\%2 Fs11165-012-9304-3\#/page-1
Study of remote lab use by undergraduate students, indicating a need for realism such as live video of the lab:

Sauter, M, Uttal, D. H., Rapp, D. N., Downing, M. \& Jona, K. (2013). Getting real: the authenticity of remote labs and simulations for science learning. Distance Education, 34(1), 37-47. groups.psych.northwestern.edu/uttal/ documents/Sauteretal2013.pdf

Study of introduction of remote lab use in a Brazilian school, indicating importance of access on mobile devices and teacher development: Simão, J. P. S., de Lima, J. P. C., Rochadel, J. B. daS. (2014). Remote Labs in Developing Countries: An Experience in Brazilian Public Education. In proceedings of IEEE 2014 Global Humanitarian Technology Conference, 1-13 October 2014, San Jose, CA. 99-105. ieeexplore.ieee.org/stamp/stamp. jsp?arnumber $=6970267$

Report on the status of science labs in US high schools:

Singer, S. R., Hilton, M. L. \& Schweingruber, H. A. (Eds.) (2005). America's Lab Report: Investigations in High School Science.

Washington DC: National Academies Press. www.nap.edu/catalog/11311/americas-labreport-investigations-in-high-school-science

Review of guidance for supporting student use of online and remote labs:

Zacharia, Z. C., Manolis, C,. Xenofontos, N., de Jong, T., Pedaste, M., van Riesen, S. A. N., Kamp, E. T., Mäeots, M., Siiman, L., \& Tsourlidaki, E. (2015). Identifying potential types of guidance for supporting student inquiry when using virtual and remote labs in science: a literature review. Educational Technology Research and Development, 63(2), 257-302.

link.springer.com/article/10.1007\%2 Fs11423-015-9370-0\#/page-1 


\section{Embodied learning}

Making mind and body work together to support learning

Potential impact: medium

Timescale: long (4+ years)

Embodied learning comes from self-awareness of one's own body - its movements, biomedical measurements, limits and interactions with the world. Whilst it is essential for some forms of learning, such as playing a new sport or learning to drive a car, it also relates more generally to how the body shapes and conditions our cognitive learning. As we move through environments and interact with people and objects, our bodies and limbs fit in with the surroundings. We continually adjust, without conscious awareness, to the terrain as we move about, the surrounding temperature, the objects and people we touch. The environment provides opportunities for action (affordances) that our bodies detect and act upon by walking, running, hearing, seeing, touching, smelling, tasting. Learning happens when this continuous process of action and reaction comes to our attention. This can happen when the body does not fit neatly into the environment (perhaps it stumbles at a concealed step), or we are learning to perform a physical activity such as dancing, or we are shaping the environment to our needs (for example, by drawing, painting, sculpting or building).

\section{How embodiment affects learning}

It follows that physical acts - such as using a pen, pencil or brush to write and draw - affect how we learn. When writing or drawing by hand rather than typing on a keyboard we are able to cross out, add notes and draw diagrams alongside the text. The process of creating a text, mathematical solution, or drawing can be made visible to other learners or teachers. Students may use their workings and crossings out to gain insight into thinking processes, see gaps in knowledge, and suggest ways to improve the technique, perhaps by adding notes or drawings. These acts of mind and body working in harmony to express the process of thinking can be powerful aids to teaching and learning. Children show their working of subtraction problems by crossing out and adding figures; poets leave traces of their creative processes on the written manuscript.

\section{f Embodied learning comes from self- awareness of one's own body $y$}

Devices with pen or touch input can capture these pen strokes and replay them to provide an animation of problem solving. Teachers can create 'worked example' videos to demonstrate the process of solving a mathematical problem or to explain a concept by drawing and adding to a diagram. New technologies that are sensitive to pressure of touch allow more subtle ways of communicating the process of drawing and painting. However, these are digital imitations of the flow of ink and the colour of pigment. Paints create colour by reflecting ambient light, while computer screens transmit and filter light.

Technology can add value when communicating through gesture, with interactive surfaces such as tabletops (large multi-touch computer screens, presented horizontally at waist height) supporting individual and collaborative work. Using these interactive tools, learners can manipulate digital information directly with their fingers, feet or other bodily movements.

Gestures enable people to communicate by indicating or moving real or virtual objects, working together to produce shapes and patterns, and creating music and dance. Gestures may also have 
a fundamental role in children's development of mathematics and science, as they rotate shapes, organise things into categories, pour liquids, and move objects into alignment or set them in motion. The speech children use with adults in performing these actions, such as 'push' and 'pour', forms the basis for scientific language.

In some instances, tabletops are better for supporting learning than using a mouse and cursor on screen, as there is a more direct route between a person's cognitive intent and their physical movements. For example, in the European JuxtaLearn project, groups of school children worked together on digital tabletops to produce joint explanations of 'tricky topics' in science and mathematics. Using their hands, they were able to move and re-order on the large screen boxes of text that represented the structure and content of their explanations. As they explained their actions to others, and engaged physically with the text, they probed their own understanding of the scientific concepts.

\section{New developments in embodied learning}

Mobile and wearable devices now contain a range of different sensors that can provide information not only about our external environment (including ambient temperature, light levels and location) but also about an individual's physical and biological data (such as the number of steps walked over

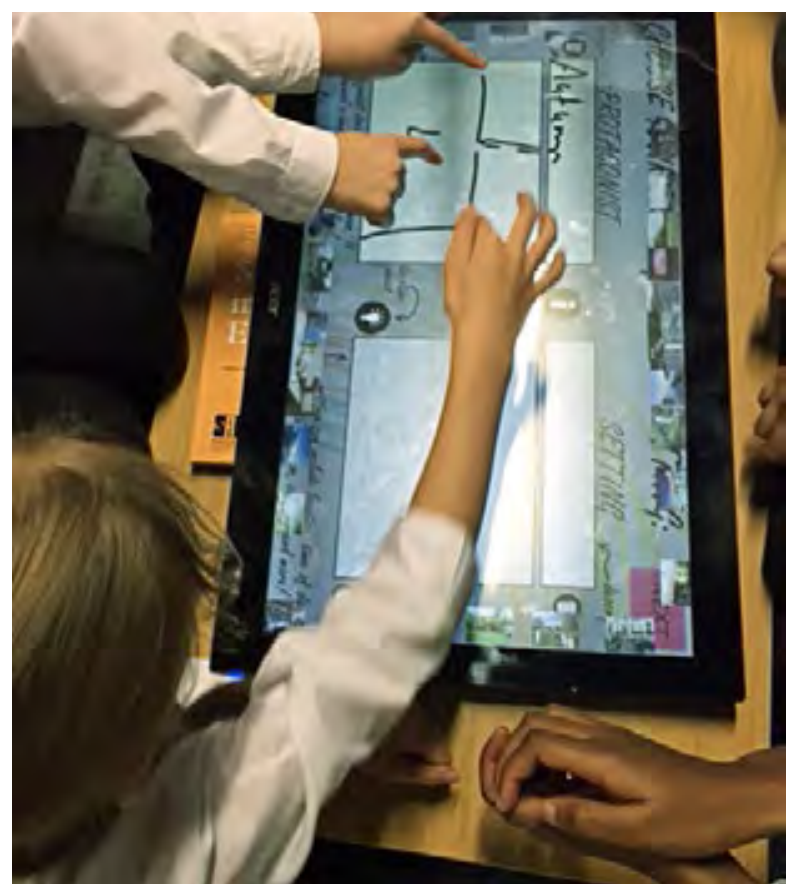

Children interacting with a tabletop display on the JuxtaLearn project a certain time period, heart rate, or even blood oxygen levels). These data, sometimes referred to as 'the quantified self', can be applied to help us learn about ourselves, to improve fitness and diagnose bodily illnesses and weaknesses.

\section{Pros and cons of embodied learning}

Embodied learning can produce new insights by generating data that are personal and directly relevant. It may also offer new ways to engage with learning that prove appealing to some learners. However, unless it is implemented thoughtfully it will present accessibility challenges, in particular to those who have physical disabilities and those who experience difficulties with physical exertion or complex bodily movements. This may result in frustration or embarrassment for those learners, leading to reduced motivation and engagement.

Other effects on motivation have been identified. Some people have reported that they find personal fitness monitors addictive, so they are reluctant to exercise when not wearing them, feeling that such activity is wasted. They have also reported feeling 'controlled' by the device, even though they experience feelings of happiness, satisfaction, pride and increased motivation when they reach their daily targets.

\section{Conclusion}

Embodied learning suggests that learning occurs not only in the mind, but also through existing and new practices engaged in by our physical bodies. Using bodily actions when learning new content may result in deeper, longer-lasting memory traces and, in some cases, higher test scores and increased knowledge retention.

New developments in wearable and implanted technologies, including internal microchips and digital tattoos are likely to provide even greater amounts of personal data about physical movements and physiology. However, these possible extensions or enhancements to minds and bodies will not always be a comfort or a benefit. It is clear that we need carefully to consider the potential negative impacts on learning, such as demotivation and intrusion, before we engage with these developments more extensively. 


\section{Resources}

Khan Academy teaching by worked example videos:

www.khanacademy.org

SMALLab Learning advancing embodied learning in schools and museums:

smallablearning.com/embodied-learning/

Duus, R. \& Cooray, M. (2015). How we discovered the dark side of wearable fitness trackers, The Conversation, June 19, 2015. theconversation.com/how-we-discovered-thedark-side-of-wearable-fitness-trackers-43363

McNerney, S. (2011). A Brief Guide to Embodied Cognition: Why You Are Not Your Brain. Scientific American Blog Network, November 4, 2011. blogs.scientificamerican.com/guest-blog/abrief-guide-to-embodied-cognition-why-youare-not-your-brain/

Evidence from gestures by teachers and learners that mathematical knowledge is embodied:

Alibali, M. W. \& Nathan, M. J. (2012). Embodiment in mathematics teaching and learning: evidence from learners' and teachers' gestures, Journal of the Learning Sciences, 21(2), 247-286.

website.education.wisc.edu/ mnathan/

Publications_files/2012_Alibali\%26Nathan_ JLS_Embodiment_Instruction_Gesture.pdf
Evans, M. A. \& Rick, J. (2014). Supporting learning with interactive surfaces and spaces. In J. M. Spector et al. (Eds.), Handbook of Research on Educational Communications and Technology (fourth edition). New York: Springer-Verlag. 689701.

A related paper can be downloaded from edutech.uni-saarland.de/uploads/330/issicls2012.pdf

Reflection on the body's role within teaching and learning, based on observation of a school science teacher:

Latta, M. M. \& Buck, G. (2008). Enfleshing embodiment: 'falling into trust' with the body's role in teaching and learning, Educational Philosophy and Theory, 40(2), 315-329.

www.tandfonline.com/doi/pdf/10.1111/j.14695812.2007.00333.x

Review of literature from anthropology, linguistics, psychology and education:

Roth, W-M. (2001). Gestures: their role in teaching and learning. Review of Educational Research, Fall 2001, 71(3), 365-392. www.cogsci.ucsd.edu/ nunez/COGS160/ Roth_PS.pdf 


\section{Adaptive teaching}

\section{Adapting computer-based teaching to the learner's knowledge and action}

\section{Potential impact: medium \\ Timescale: long (4+ years)}

Adaptive teaching refers to the ways in which computer applications can analyse data from learning activities to provide learners with relevant content, to sequence their learning activities, to address gaps in their knowledge, and to accelerate their learning. The term can also apply to software that helps teachers to adapt their classroom activities based on responses from students. Adaptive teaching is distinct from interactive learning, which takes place when a learner uses technology that responds to input but does not adapt to the specific characteristics of the learner. Adaptive teaching products have been developed for use in classrooms, in workplaces and at home.

\section{ff A teacher in a classroom} cannot provide personal assistance to each student, yet giving such
immediate feedback may improve student performance $y$

Learners have differences in their knowledge, experience, and preferences for studying. Even when a school screens learners using pre-requisite courses, students will differ in proficiency and confidence. For example, consider two learners who have passed high school algebra and join the same college mathematics course. One learner has a passion for algebra, whereas the other considers it an exercise required to get a degree. Their attitudes and skills in the class may be quite different: the first may be bored, wanting extra challenge, while the other may be anxious and unprepared. Adaptive teaching attempts to adjust to differences in background knowledge and experience, providing ways for learners to cope. Such technology also can provide just-intime feedback by responding to recent actions, to correct an error or offer a hint. A teacher in a classroom cannot provide personal assistance to each student, yet giving such immediate feedback may improve student performance.

\section{Learning at one's own pace, visualizing learner differences}

Picture a student who is taking a course in college that uses adaptive technology software. The student thinks some parts of the curriculum are interesting and some are boring. Instead of being forced to do the parts that she considers boring, whenever the course reaches that point she can choose her own sequence in which to study the material. When she is tested at the end of each section, the more she is stuck on a problem the more hints she receives. Once she understands easier problems, she is given more difficult ones. The computer suggests weaker areas she should review. At the end of the day, the teacher views the performance of each student and uses feedback from the system to find that a large group of students had trouble with certain questions, so plans either to re-teach those concepts in class the next day, or to split the students into small groups to discuss specific problems, as suggested by the software.

\section{Adaptive teaching technologies}

The number of adaptive learning products is expanding at a rapid pace. Some educators see adaptive approaches as providing flexible study options to students, particularly those struggling in remedial and general education courses or fully 


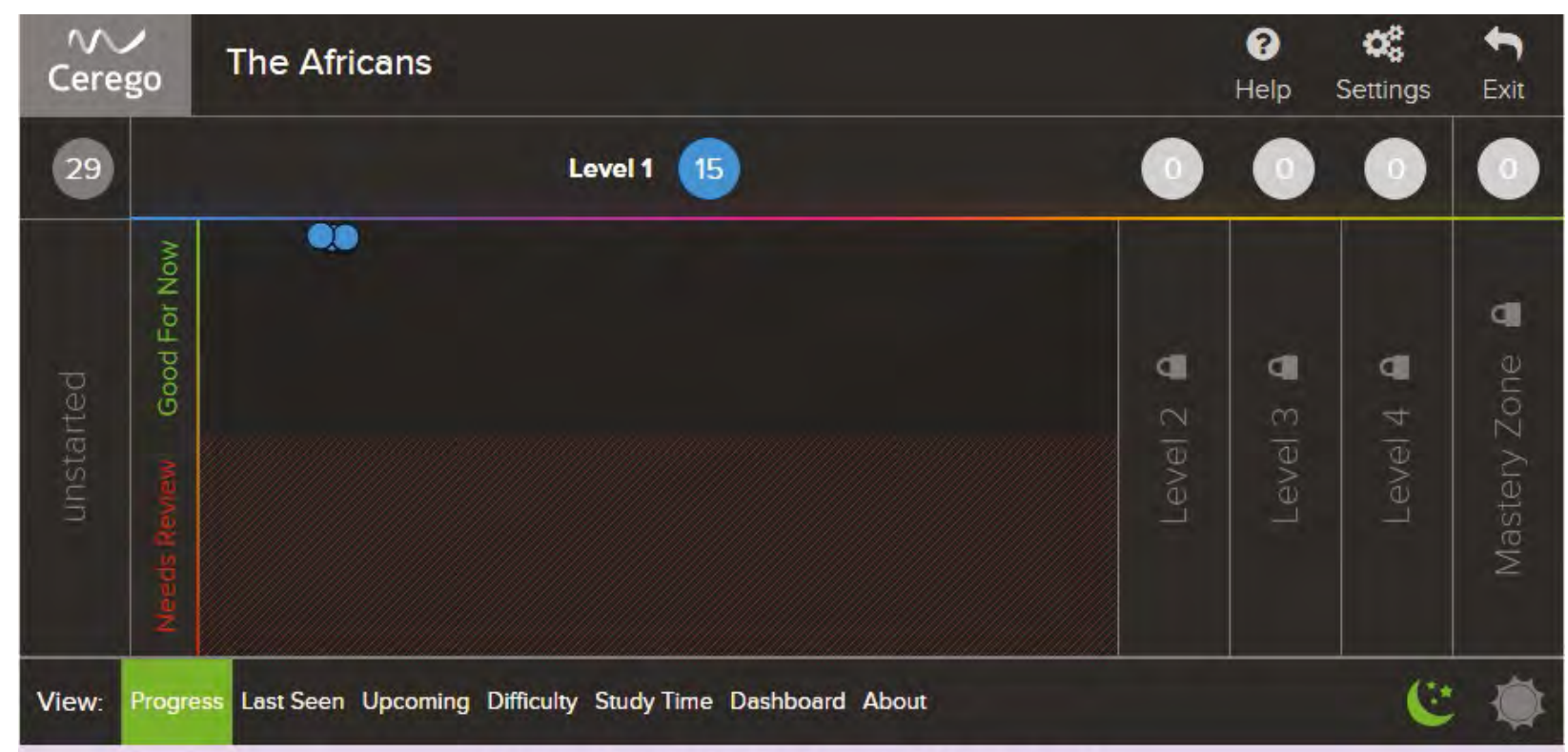

You got a progress bump! Keep it up!

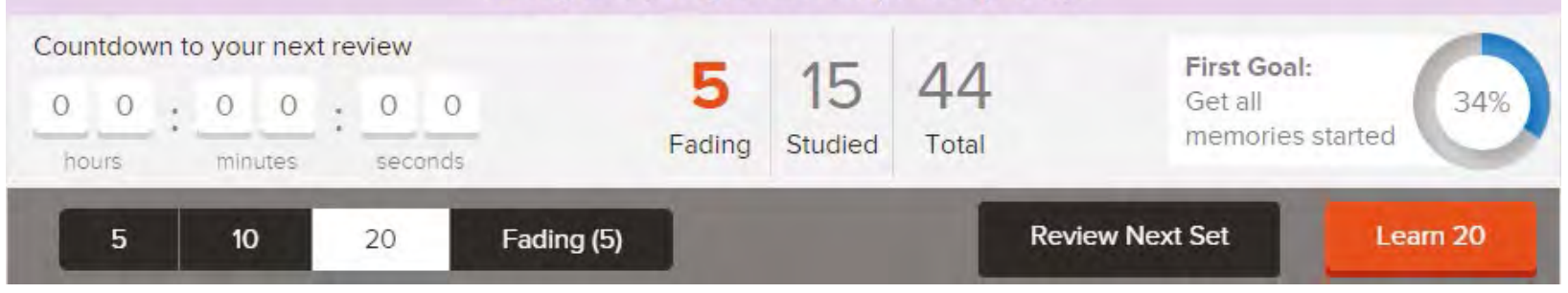

Learner dashboard from the Cerego memorisation system

online classes. Many of these products have their roots in 1980s research into 'intelligent tutoring systems'. These were developed primarily to support mathematics teaching, but new products address a broad range of subjects including English, psychology, economics and biology. Recent research into adaptive technologies is expanding beyond cognitive learner data by responding to learners' moods and brain activity.

Adaptive products are designed and sequenced by professional software designers and educational technologists. Pearson's MyLab and Mastering products include many subjects: biology, chemistry, engineering, psychology, writing, culinary sciences, oceanography and accounting. The Knewton uses psychometric tools to analyse student ability, probabilistic models to recommend the learning activities to complete next, clustering to group students by proficiency, and decay curves that model forgetting and trigger reminders to study older material. Knewton, like others such as Smart Sparrow, allows a teacher to select the lesson design and to set the range of adaptability.

Smart Sparrow provides tools to create simulations and interactive content. CogBooks enables educators either to select from previously created courses or to create their own. Its adaptive course sequence is neither a pre-set path, nor one based on test results, but it employs algorithms and machine learning methods that continuously tailor a learning sequence for each learner. It also adds learner interests to standard educational content, for example, producing mathematics problems focused on an industry that appeals to the learner.

Cerego memorisation software asks learners to set goals based on the content they need to cover and the time available to study it, then designs a daily study plan. Brainscape adapts flashcards based on a learner's confidence and available study time. Its model is informed by cognitive science research on how teaching methods should be sequenced, timed and repeated in order to store knowledge reliably in long-term memory.

Adaptivity has also been applied to massive open online courses (MOOCs) and online learning content to support learners based on their preferences for pedagogical approach. They can choose from approaches as diverse as closely mentored apprenticeship and discovery through experimentation. An interactive companion to the Mathspring math tutoring game sends encouraging messages to students and adapts the 
game difficulty depending on learner confidence, interest, frustration or excitement. Another tutoring program adapts content by monitoring brain activity and also documents workload, boredom, off-task activity and engagement.

\section{Adapting adaptive teaching}

Previous editions of Innovating Pedagogy have addressed ingredients of adaptive learning including learning analytics, dynamic assessment, and learning from gaming. Despite much progress, adaptive teaching faces barriers. The cost of developing adaptive teaching systems can be high if teaching material must be produced to match the needs and interests of different learners. Where traditional approaches require a linear string of lessons, adaptive learning requires branched packets of content at several levels to provide multiple pedagogical approaches, confront common misconceptions, and offer a battery of hints that give learners the appropriate level of challenge and support. Systems for mathematics and language teaching have been designed to generate examples and hints at varying levels of difficulty, matched to the learner's actions. They can generate exercises, detect where the student makes an error, and indicate the misunderstanding. However, the design of these tutoring systems can involve years of research into learners' misconceptions. For this reason, cost-benefit analysis and developing efficiencies around analysis and generation of content are particularly useful for the growth of adaptive teaching technologies.

\section{Resources}

Adaptive flashcards:

https://www.brainscape.com

Cerego adaptive software for learning based on spaced rehearsal for memory retention:

cerego.com

CogBooks: adaptive learning books:

pulb.cogbooks.com/ cogbooks/product/

Knewton system for teachers to create adaptive learning content:

www.knewton.com

MathSpring adaptive mathematics practice software:

mathspring.org

Report on adaptive learning for Knewton: www.knewton.com/wp-content/uploads/ knewton-adaptive-learning-whitepaper.pdf

Pearson MyLab \& Mastering adaptive tutoring products:

www. pearsonmylabandmastering.com
Smart Sparrow adaptive e-learning platform: www.smartsparrow.com

Introduction to adaptive web-based systems for education:

Brusilovsky, P., \& Peylo, C. (2003). Adaptive and intelligent web-based educational systems. International Journal of Artificial Intelligence in Education (IJAIED), 13, 159-172.

telearn.archives-ouvertes.fr/hal-00197315/ document

Design, development and deployment of a MOOC that offers five learning strategies based on learner preference:

Sonwalkar, N. (2013) The First Adaptive MOOC:

A Case Study on Pedagogy Framework and

Scalable Cloud Architecture - Part I. MOOCs

FORUM, 1, 22-29.

online.liebertpulb.com/doi/abs/10.1089/

mooc.2013.0007 


\title{
Analytics of emotions
}

\section{Responding to the emotional states of students}

\author{
Potential impact: medium \\ Timescale: long (4+ years)
}

Emotions, attention and engagement are key drivers for learning. When you decide whether to get out of bed, read a complex article, or complete a challenging task, how you feel about performing these activities will determine whether you will do them, persist and, more importantly, enjoy them.

\section{Detecting emotions}

Detection of emotions using advanced artificial intelligence approaches is an approach used in marketing and advertising. Several companies can accurately measure how people experience emotions, engagement, and attention while watching an advertisement, using only a simple webcam on a tablet, smartphone or laptop. By combining eye-tracking movements (the way a viewer's eyes focus on particular elements in a video or text) with facial expressions (such as amazement, or a shake of the head) and posture (leaning backwards or forwards), several companies can already track, trace and predict how people react to particular advertisements, where their attention is drawn, whether they like or dislike a particular scene, and whether they associate the brand with particular emotions.

Rather than running expensive expert and customer focus groups to establish whether an advert works or not, marketing companies have found that thousands of people are freely willing to share their emotional reactions to featured advertisements just by sitting behind a screen. By repositioning certain elements and scenes based
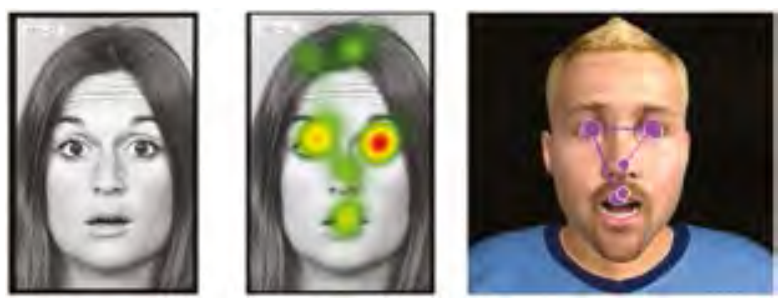

Eye-tracking study - the overlaid colours show where the viewer is looking upon the emotional reactions of volunteers, these companies adjust the way groups of people will react to emotional inputs and thereby maximise the chance that people will remember a brand positively.

In the field of the learning sciences, researchers are on the brink of a similar breakthrough. Techniques for tracking eye movements, emotions and engagement have matured over the past decade. Combining a mature discipline with relatively inexpensive ways to measure where learners watch and click in learning materials will allow researchers, teachers and learners to discover where, when, and how people learn.

While you are reading this text, you may simultaneously be keeping an eye on a chat stream, listening to Spotify, or overhearing a nearby conversation. If this text is truly engaging and interesting, your mind, eyes, and body will be fully immersed in the learning experience. In the future, using analytics of emotions, institutions will be able to track which learning materials students are following, and whether they are distracted, guessing answers to quiz tests, or really engaged in learning.

\section{Personalised learning based on emotional responses}

By combining people's clicking and typing behaviour with the areas where they focus their gaze, rich opportunities for personalised learning could become available. For example, if a student is glancing back to earlier material, it may be that the text is not sufficiently clear and he is struggling with some concepts. If he is continually looking for key terms, scrolling back and has a puzzled facial expression (or worse, is getting annoyed), the system may suggest a short video explaining key 
concepts in an engaging manner, or offer a quick help function.

\section{if analytics of emotions}

\section{can work alongside adaptive teaching to offer a more personalised learning experience $y$}

Alternatively, if the analytics indicate that a student is rapidly skimming text, she may already be already familiar with key concepts and be getting bored, so an engaging exercise might pop up, or a chat opportunity to discuss a complex problem with a peer. Thus, analytics of emotions can work alongside adaptive teaching to offer a more personalised learning experience.

While there may be valuable opportunities to link eye-tracking and emotion data with learning behaviour data, learners will only be willing to share eye movements, facial expressions and posture data with others if they perceive positive benefits. One such benefit would be that the analytic tools are able to identify learners' emotions correctly and then provide appropriate teaching and useful feedback. Even as the practical problems are solved, complex ethical and privacy challenges will need to be addressed, if learning providers wish to monitor students' emotional reactions. They will need to develop trust with learners and educators by providing accurate, adaptive learning solutions based upon actual learning emotions and needs.

\section{Resources}

Test your own emotional reactions to advertising videos:

www.affectiva.com/technology/

Project by Sidney D'Mello and Ryan Baker to detect emotions while students learn from the Newton's Playground game for physics learning: sites.google.com/site/sidneydmello/ projects\#TOC-Emotions-while-Students-Learnfrom-Newton-s-Playground

Introduction to how researchers are gathering data from many sources, including emotion, to develop computer-based tutoring systems: Koedinger, K., D’Mello, S., McLaughlin, E. A., Pardos, Z. A., \& Rosé, C. P. (2015). Data mining and education. Wiley Interdisciplinary Reviews: Cognitive Science, 6(4), 333-353.

onlinelibrary.wiley.com/doi/10.1002/wcs.1350/ abstract

Visschedijk, G. C., Lazonder, A. W., van der Hulst, A., Vink, N., \& Leemkuil, H. (2013). Modelling human emotions for tactical decision-making games. British Journal of Educational Technology, 44(2), 197-207.

publications.tno.nl/publication/100054/

RqYpW1/visschedijk-2013-modelling.pdf
Design of adaptive tutoring based on overcoming negative student emotions:

Arroyo, I., Muldner, K., Burleson, W., \& Woolf, B. P. (2014). Adaptive interventions to address students' negative activating and deactivating emotions during learning activities. In R. Sottilare, A. Graesser, X. Hu \& B. Goldberg (Eds.). Design Recommendations for Intelligent Tutoring Systems Vol 2 (pp. 79-92). Orlando, FL: U.S. Army Research Laboratory. ict.usc.edu/pubs/Intelligent $\% 20$ Tutoring $\% 20$ Support $\% 20$ for $\% 20$ Learners $\% 20$ Interacting\%20with\%20Virtual\%20Humans.pdf

Study comparing people's ability to recognise emotions on real and virtual faces:

Joyal, C. C., Jacob, L., Cigna, M-H., Guay, J-P. \& Renaud, P. (2014). Virtual faces expressing emotions: an initial concomitant and construct validity study. Frontiers in Human Neuroscience, 8(787). Published online. www.ncbi.nlm.nih.gov/pmc/articles/ PMC4179743/ 


\title{
Stealth assessment
}

\section{Unobtrusive assessment of learning processes}

\author{
Potential impact: medium \\ Timescale: long (4+ years)
}

As people play modern computer games exploring simulated worlds, combatting foes and overcoming challenges - the computer software monitors their progress, changing the game world, spawning new enemies and setting increasingly difficult problems. This approach of continually tracking a person's progress while providing immediate automated responses has been termed stealth assessment and it is starting to be applied to educational games and simulations.

Stealth assessment extends adaptive teaching by making continual adjustments to a simulated environment, rather than selecting a path or exercise based on diagnosis of a learner's knowledge and misconceptions. The assessment is embedded within the flow of the game and the student is likely to be unaware that this dynamic process of monitoring and response is taking place.

\section{\& The pedagogy that underlies stealth}

\section{assessment is that of competency learning}

The term was first used by Valerie Shute in 2005 to describe the automated assessment process in a system named Smithtown to teach principles of microeconomics (for example, the laws of supply and demand) while students explored a simulated world and altered variables such as the price of coffee and the incomes of inhabitants. The aim was to teach students how to engage in inquiry learning by forming hypotheses and testing predictions. The software used methods from artificial intelligence to monitor and analyse the students' actions, providing them with feedback to support their inquiry skills.

\section{Principles and theories of stealth assessment}

The key principles of stealth assessment are that:

- the software analyses interactions of students within a computer game or simulation

- the system continually adjusts the structure of the game to support learning, for example by offering new challenges of a form and level matched to the student's performance

- the system maintains the flow of the game, so that the teaching and assessment are part of the game rather than added layers

- the system builds a dynamic model of the learners to indicate their abilities and competences

- it is intended to reduce learners' anxiety about taking tests by blurring the distinction between assessment and learning, while carrying out accurate diagnosis.

The pedagogy that underlies stealth assessment is that of competency learning. The teacher (or, in the case of stealth learning, the computer) estimates what the student knows and can do, continually providing tasks and assessment that are matched to the student's competency. To do this the teacher, or teaching system, must diagnose how the student is performing on specific problems and then infer levels of competency across a network of skills. The objective is to detect the student's problemsolving skills involving knowledge, comprehension and application while also uncovering the higherlevel abilities of creativity and critical thinking.

The pedagogy that underlies stealth assessment is that of competency learning. Automated collection of the assessment data relies on matching the pedagogy of competency learning with methods of computer game design including: setting goals 
to be achieved, managing conflict or challenge, providing continual responses, creating a compelling game story, designing a believable simulated environment, and offering meaningful interactions within the environment.

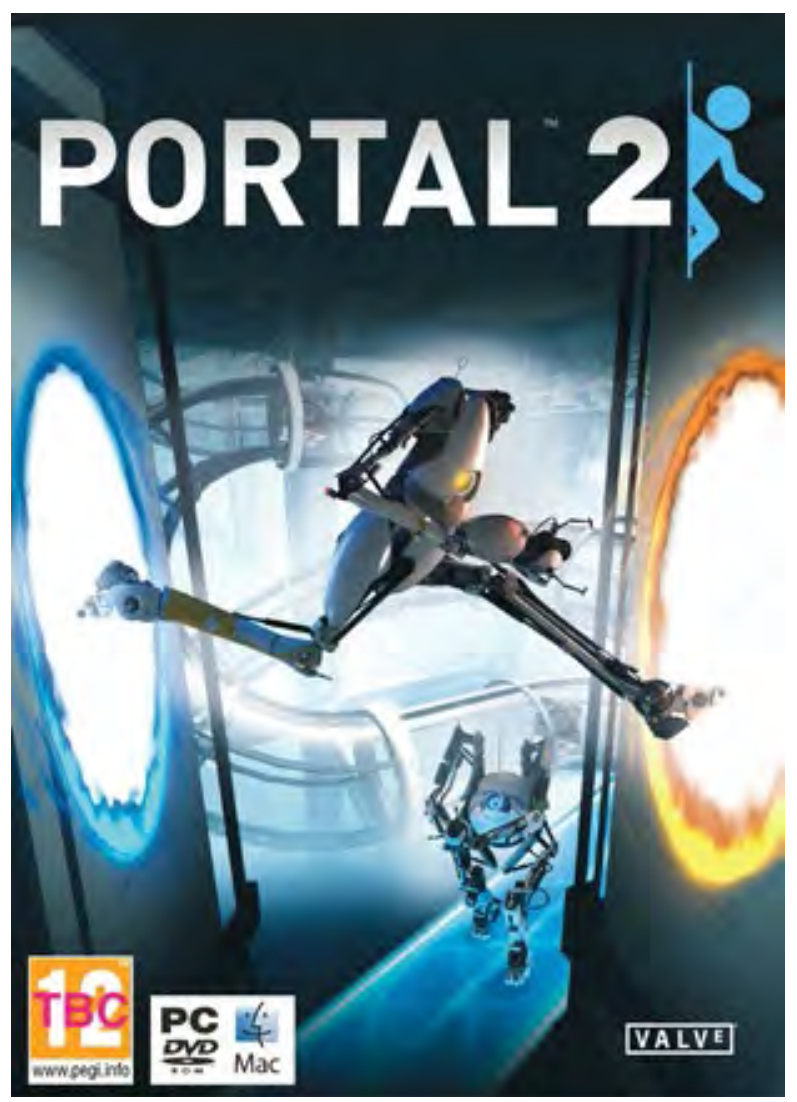

Cover art for Portal 2 game

A successful method of developing stealth assessment games is through Evidence-Centred Design. First, the educational game designer needs to determine what knowledge, skills and competencies will be assessed, so that they can be built into the gameplay. These attributes cannot be assessed directly (since the game has no direct way of knowing what the student is thinking and the stealth approach precludes setting explicit tests of knowledge), so the designer has to work out which behaviours and interactions will provide evidence of a player's knowledge, skills and competencies. Then the game is constructed to provoke these activities, with measures of success and failure in performance as the learner undertakes a mission or solves a game problem. These measures are interconnected to form a network of probabilities that the learner has gained the desired skill or reached the required level of competency.

\section{Examples of stealth}

\section{assessment}

An example of a computer game that employs stealth assessment is Portal 2, developed by Valve Corporation. The player takes the role of Chell, who has to explore an advanced science laboratory, realised as a complex mechanised maze, and find an exit door by using a set of tools. An educational aim is for the user to learn aspects of physics, to gain visual spatial skills and to develop critical thinking abilities.

Another, very different, example of stealth assessment can be found in the TAALES tool. This analyses the lexical properties of students' essays (such as word frequency and use of academic language) to assess the students' vocabulary knowledge. Stealth assessment of student essays with TAALES is being coupled to a system that helps the students to improve their essay-writing skills.

\section{Opportunities and challenges}

Stealth assessment works best when the assessment strategies, the game and the simulated world are all developed together through a process of evidence-centred design that applies not only to the assessment but also the gameplay (so that the game elements are included to stimulate engagement and learning). A less successful approach is to add dynamic assessment to an existing game or simulation.

Stealth assessment techniques can give learners immediate feedback on their actions and also provide teachers with information on how each learner is developing skills of inquiry, critical thinking, decision-making and creativity. This work is at an early stage and it is not yet clear whether the methods of stealth assessment need to be developed afresh for each game and topic, or whether general methods of design can be adopted.

The term 'stealth assessment' evokes attempts by teachers and game designers not only to monitor learners' actions but also to infer their motivations, competences and limitations, whether or not 
the learners are willing or give permission. For research projects, these systems can, and should, be developed within strict ethical guidelines that include telling the learners how they are being monitored, how the information will be used, and gaining informed and willing consent from the participants. But stealth assessment is already being embedded into commercial games and might, for example, be used without players' knowledge to assess insurance risks.
Stealth assessment offers engaging ways to teach competencies such as creativity, problem solving, persistence and collaboration, by incorporating dynamic assessment and feedback into computer games. The methods need to be introduced with care and sensitivity, but early results show promise in combining the engagement of simulation games with the diagnostic power of dynamic assessment.

\section{Resources}

Portal2 game:

www.thinkwithportals.com

Study of TAALES for essay assessment: Allen, L. K. \& McNamara, D. S. (2015). You Are Your Words: Modeling Students' Vocabulary Knowledge with Natural Language Processing Tools. In proceedings of the 8th International Conference on Educational Data Mining, 26-29 June 2015, Madrid, Spain. www.educationaldatamining.org/EDM2015/ proceedings/full258-265. pdf

Evidence-centred design:

Messick, S. (1994). The interplay of evidence and consequences in the validation of performance assessments. Educational Researcher, 23(2), 13-23.

onlinelibrary.wiley.com/ doi/10.1002/j.2333-8504.1992.tb01470.x/pdf
Introduction to stealth assessment:

Shute, V. J. (2011). Stealth Assessment in computer-based games to support learning. In S. Tobias and J. D. Fletcher (Eds.) Computer Games and Instruction, Charlotte: Information Age Publishing. 503-524.

http://myweb.fsu.edu/vshute/pdf/shute $\% 20$ pres_h.pdf

Comprehensive report on stealth assessment: Shute, V. \& Ventura, M. (2013). Stealth assessment: measuring and supporting learning in video games. Cambridge, MA: MIT Press. http://myweb.fsu.edu/vshute/pdf/Stealth_ Assessment.pdf 


\section{Innovating \\ Pedagogy 2015}

Exploring new forms of teaching, learning and assessment, to guide educators and policy makers

\section{Open University Innovation Report 4}
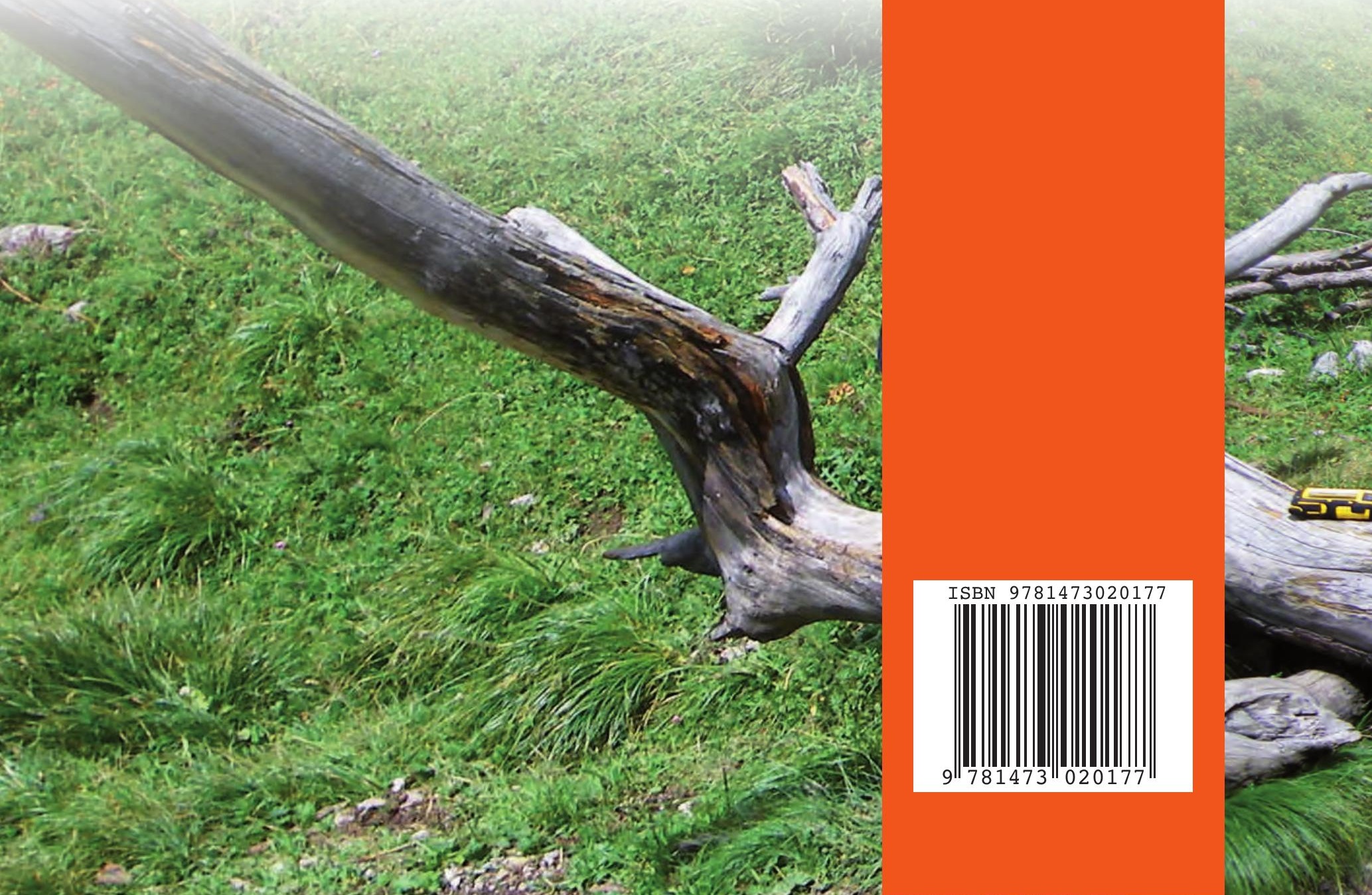